\title{
Development of the Embryonic Neuromuscular Synapse of Drosophila melanogaster
}

\author{
Kendal S. Broadie and Michael Bate \\ Department of Zoology, University of Cambridge, Cambridge CB2 3EJ, United Kingdom
}

We have examined the embryonic development of an identified neuromuscular junction (NMJ) of Drosophila melanogaster using whole-cell patch-clamp and a variety of physiological and morphological techniques. Synaptic current at the embryonic NMJ is carried through a large-conductance (200 pS) L-glutamate receptor. Early synaptic communication is characterized by frequent, brief $(<10 \mathrm{msec}$ ) currents carried through few $(1-10)$ receptors and relatively rare, prolonged currents (up to seconds) of similar amplitude. The brief currents have a time course similar to the mature larval excitatory junction currents (EJCs), but the prolonged currents are restricted to early stages of synaptogenesis. The amplitude of EJCs rapidly increases, and the frequency of the prolonged currents decreases, after the initial stages of synaptogenesis. Early prolonged (seconds), nonspiking synaptic potentials are replaced with rapid $(<0.10 \mathrm{sec}$ ), spiking synaptic potentials later in development. The early synapse appears tenuous, easily fatiguable, and with inconsistent communication properties.

Synaptogenesis can be divided into a sequence of progressive stages. (1) Motor axon filopodia begin neurotransmitter expression and concurrent exploration of the myotube surface. (2) Myotubes uncouple to form single-cell units soon after motor axon contact. (3) A small number of transmitter receptors are homogeneously displayed on the myotube surface immediately following myotube uncoupling. (4) Endogenous transmitter release from pioneering growth cones is detected; nerve stimulation elicits postsynaptic EJC response. (5) Motor axon fllopodia and transmitter receptors are localized to the mature synaptic zone; filopodial localization is complete in advance of receptor localization. (6) A functional neuromuscular synapse is formed; endogenous muscular activity begins; nerve stimulation leads to muscle contraction. (7) Morphological presynaptic specializations develop; synapse develops mature morphology. (8) A second motor axon synapses on the myotube at the pre-established synaptic zone. (9) Vigorous neuromuscular activity, characteristic of larval locomotory movements, begins. (10)

\footnotetext{
Received Mar. 2, 1992; revised July 13, 1992; accepted July 14, 1992.

We are grateful to Dr. Roger Hardie for excellent advice, technical and otherwise throughout the course of this study. We thank Dr. Jeremy Skepper and Anthony Burgess for technical assistance with the SEM, Drs. Douglas Curric and Helen Skaer for advice with culturing techniques, and Dr. David Sattelle for the gift of Argiotoxin-636 used in this study. Drs. Helen Skaer, Roger Hardie, and Rachel Drysdale critically read earlier versions of the manuscript. This work was supported by an Oliver Gatty studentship and AFCU scholarship to K.S.B. and by grants from the Hassleblad Foundation and Wellcome Trust to M.B.

Correspondence should he addressed to Kendal Broadie, Department of Zoology, Downing Street, Cambridge CB2 3EJ, UK.

Copyright (C) 1993 Society for Neuroscience $0270-6474 / 93 / 130144-23 \$ 05.00 / 0$
}

A second stage of receptor expression begins and continues through the end of embryogenesis.

In general, Drosophila neuromuscular synaptogenesis appears similar to neuromuscular synaptogenesis in known vertebrate preparations. We suggest that this system pro. vides a model for synaptogenesis in which investigation can be readily extended to a genetic and molecular level.

IKey words: Drosophila, neuromuscular junction, synaptogenesis, embryo, glutamate receptors, invertebrate embryogenesis]

During the development of the neuromuscular junction (NMJ), many elements must interact to form an efficient communication system: the motor neuron must find the appropriate target muscle and arborize correctly on that muscle, signaling mechanisms (i.e., transmitter synthesis, vesicle synthesis and localization, excitation-transmitter release coupling, etc.) must develop in the presynaptic nerve terminals, and receptive mechanisms (i.e., transmitter receptor synthesis and localization, excitation-contraction coupling, etc.) must develop in the postsynaptic muscle. All of these developmental processes must be temporally and spatially coordinated to generate the precise, stereotyped, mature NMJ. We wish to know how this developmental interaction is orchestrated at the cellular, molecular, and genetic level.

Our understanding of chemical synapses in general (reviewed in Saltpeter, 1987), and synapse development in particular (reviewed in Bennet, 1983; Sanes and Poo, 1988), relies heavily on the vertebrate NMJ as a model. Studies for several decades have generated a vast store of information, characterizing the mature synapse and describing the phenomenology of its development. We know, for example, that initially diffusely distributed $\mathrm{ACh}$ receptors (AChRs) become localized to presynaptic release sites during embryonic development (Anderson and Cohen, 1977). We know that presynaptic terminals make precise contacts with the muscles from the onset and that such contacts are likely mediated through preferential adhesion mechanisms (Landmesser et al., 1990). We know the time course and many characteristics of the physiological and morphological NMJ development in several vertebrate systems, both in vivo (Kullberg et al., 1977; Dennis et al., 1981; Lupa and Hall, 1989) and in vitro (Poo et al., 1985; Evers et al., 1989). On the other hand, much less is known about formative interactions. In the best-studied case, AChR localization has been argued to be induced by the motor neuron terminals, via electrical activity (Rochlin and Peng, 1990), the release of specific signaling molecules (Shadiack and Nitkin, 1991; Wallace, 1991; Lieth et al., 1992), or possibly both. This information has been the result of a great deal of effort by many laboratories, but is still only 
the initial step in unraveling one of the multitude of complex interactions underlying neuromuscular synaptogenesis.

A major obstacle to understanding dynamic biological processes such as synaptogenesis is that it is usually impossible to study the critical formative events directly as they happen, a difficulty that is exacerbated in the complex vertebrate embryo. For this reason, most experimental investigations of vertebrate synaptogenesis have had to be done in the simplified environment of culture systems (Bursztajn et al., 1989; Evers et al., 1989; Rochlin and Peng, 1990). Even if this were not the case, and we note the recent elegant in vivo analyses of several groups (Balicegordon and Lichtman, 1990; Wigston, 1990), extending our understanding of synaptogenesis to a molecular or genetic level would be difficult in the vertebrate preparations presently employed. Due to difficulties inherent in vertebrate systems, little is known about molecular interactions during synapse development and nothing is known about the genes controlling these processes.

The embryonic NMJ of Drosophila melanogaster is an attractive alternate system to expand and extend our understanding of neuromuscular synaptogenesis and synapse formation in general. The mature Drosophila NMJ shares the experimental benefits of the vertebrate system and has been extensively characterized (Jan and Jan, 1976a,b; Johansen et al., 1989a). Furthermore, the Drosophila NMJ has been employed for many years to generate and examine mutations in chemical synapse function (reviewed in Ganetzky and $\mathrm{Wu}, 1986$; Zhong and $\mathrm{Wu}$, 1991). In addition, Drosophila embryogenesis is rapid (less than a day) and the embryonic NMJ can be experimentally manipulated in vivo throughout the course of its development. Thus, the intact embryo has many of the advantages of simple systems such as those derived from cultured muscle cells. Several groups (Johansen et al., 1989b; Halpern et al., 1991; Sink and Whitington, $1991 \mathrm{a}, \mathrm{b})$ have already exploited these benefits to detail the morphological development of the NMJ in the Drosophila embryo and have thus laid the groundwork for a more detailed analysis. Finally, and most importantly, proven Drosophila techniques provide a means of extending our understanding of chemical synaptogenesis to a genetic and molecular level.

It is the purpose of this study to document the normal dcvelopment of the Drosophila embryonic NMJ and so establish a framework for subsequent experimental and genetic dissection. At the outset of this work, very little was known of synaptogenesis at the invertebrate NMJ, and it was not clear whether the basic processes underlying vertebrate NMJ formation have been conserved. We have used whole-cell patch-clamp analysis of the developing myotubes, in combination with a variety of morphological and physiological techniques, to examine the normal development of an identified NMJ during Drosophila embryogenesis. In this study, we are able to show that much of Drosophila NMJ synaptogenesis parallels processes in known vertebrate preparations. We are encouraged by these findings and expect that this system will be a useful model for vertebrate NMJ formation and chemical synaptogenesis in general.

\section{Materials and Methods}

Fly stocks. The wild-type Drosophila melanogaster strain Oregon- $\mathrm{R}$ was used throughout. Occasionally, a mutant strain (foreclosed; $f c^{244 \mathrm{~b}}$, which fails to close dorsally and has a weak cuticle, was used to facilitate study of late embryonic stages when a tough wild-type cuticle makes dissection difficult. Experiments with the mutant were confirmed using dissected and/or cultured wild-type Oregon-R embryos.
Preparation. Breeding flies were maintained on apple juice agar plates at $25^{\circ} \mathrm{C}$ and allowed to lay eggs overnight. The eggs were collected, dechorionated in commercial bleach, and placed in a plastic culture dish for staging. Embryos were staged by morphological criteria (CamposOrtega and Hartenstein, 1985; K. S. Broadie, unpublished observations) to narrow $\left(<15 \mathrm{~min}\right.$ at $25^{\circ} \mathrm{C}$ ) well-defined developmental time windows and allowed to continue development to the desired stage. Three time windows were used routinely: (1) narrow ventral furrow of gastrulation [2:50-3:00 hr after egg laying (AEL)], (2) completion of germ band retraction (8:45-9:00 hr AEL), and (3) first gut constriction (two-part gut; 12:45-12:55 hr AEL). Embryos were staged from the closest window; for synaptogenesis stages, almost entirely from the first gut constriction. All development times are reported in hours AEL at $25^{\circ} \mathrm{C}$ and are displayed as decimals. Under these conditions, embryogenesis lasts $21 \pm 1 \mathrm{hr}$.

All experiments were performed on dissected whole-embryo preparations. Younger embryos ( $<17 \mathrm{hr}$ AEL) were transferred to polylysinecoated coverslips under normal saline, removed from the vitelline membrane, cut along the dorsal midline with a glass electrode, and attached to the coverslip surface with a gentle stream of saline. Older embryos $(>17 \mathrm{hr}$ ) were transferred to coverslips coated with a thin layer of Sylgard under normal saline, cut along the dorsal midline with a fine metal blade, and pinned to the Sylgard with glass pins. The gut was then removed, exposing the ventral nervous system, peripheral nerves, and somatic musculature. All experiments were performed at the identified neuromuscular junction (presynaptic motor neuron RP3; Sink and Whitington, $1991 \mathbf{a}, \mathbf{b}$ ) of the large ventral interior-longitudinal muscle (muscle 6; Bate, 1990) in anterior abdominal segments A2-A4.

Whole embryo culture and recording solutions. The late embryo $(>17$ $\mathrm{hr} A E L$ ) has a tough cuticle that makes dissection and attachment to conventional substrates difficult. As a result, we have cultured dissected whole embryos from earlier, more amenable stages (Broadie et al., 1992). Embryos were dissected at $16 \mathrm{hr}$ AEL (as above) and cultured in a small drop (20 $\mu$ l) of modified M3 (MM3) medium (Shields and Sang, 1977; Currie et al., 1988) in a humid chamber at $25^{\circ} \mathrm{C}$. The MM3 culturing medium was similar to previously published reports, with the following exceptions/additions: (1) the glutamate salts were replaced with equimolar aspartate salts, (2) $0.125 \mathrm{IU} / \mathrm{ml}$ insulin (Sigma) was added, and (3) $2 \%$ fetal calf serum (Sigma), filtered but not heat inactivated, was added. Under these conditions, the embryo preparations appeared to develop normally as judged by all available morphological, immunohistological, and physiological criteria, with the exception that the tracheas fail to fill with air. All experiments with cultured preparations were independently confirmed with freshly dissected embryos.

All physiological recordings were performed with the same normal fly salines. The bath, which was oxygenated, consisted of (in mmol/ liter) $135 \mathrm{NaCl}, 5 \mathrm{KCl}, 4 \mathrm{MgCl}_{2}, 2 \mathrm{CaCl}_{2}, 5 \mathrm{TES}$ (N-tris[Hydroxymethyl]methyl-2-aminoethane sulfonic acid), and 36 sucrose. The intracellular solution consisted of (in mmol/liter) $120 \mathrm{KCl}, 20 \mathrm{KOH}, 4 \mathrm{MgCl}_{2}$, 5 TES, 5 EGTA, $0.25 \mathrm{CaCl}_{2}, 4$ ATP, 4 GTP, and 36 sucrose. The pH of all solutions was buffered at 7.15.

Electrophysiology: whole-cell patch-clamp techniques. For physiological experiments, the preparation was placed in a small perspex recording chamber and viewed in transmitted light with a compound microscope (Micro Instruments Ltd.) fitted with differential interference contrast (Nomarski) optics and a $40 \times$ water-immersion lens. Whole-cell recordings were made at room temperature $\left(\mathrm{RT}\right.$ : $\left.18-22^{\circ} \mathrm{C}\right)$ with patch pipettes pulled (Narishige patch-electrode puller) from borosilicate glass (fiber filled) with tips fire polished to final resistances of 5-8 M 2 . Wholecell recordings were achieved using standard patch-clamp techniques (Hamill et al., 1981; Marty and Neher, 1983). Signals were amplified using an Axopatch-1D (Axon Instruments) patch-clamp amplifier, filtered with an 8-pole Bessel filter at 2-5 Hz, and either sampled on line or stored digitally on a modified digital audio tape recorder (Sony) for later analysis. Data were analyzed using pCLAMP 5.51 software (Axon Instruments) on a Viglen III/ $33 \mathrm{MHz}$ computer.

Seal resistances were typically $>10 \mathrm{G} \Omega$. Whole-cell configuration was achieved easily with slight suction, and input resistance of the myotube was in the range of 200-500 M . With maximum currents less than 1 $\mathrm{nA}$, series resistance errors (total current $\times$ series resistance) are kept to reasonable levels (usually $<10 \mathrm{mV}$ ) and were not corrected. Myotubes with these characteristics, average diameter of $12 \mu \mathrm{m}$, and average length of $36 \mu \mathrm{m}$ should show reasonable space clamp. Series resistances were taken from the series resistance control on the amplifier and monitored throughout the experiment; typical values ranged from 10 to $25 \mathrm{M} \Omega$. 
Cell capacitances were between 7 and $14 \mathrm{pF}$, increasing with developmental age, generating clamp time constants $\left(R_{\text {scrics }} \times C_{\text {cell }}\right)$ averaging less than $0.25 \mathrm{mscc}$.

Perforated patch-clamp recording. We were concerned that dilution of the cellular cytoplasm, especially with long-time-course whole-cell recordings (up to $1 \mathrm{hr}$ ) or during sensitive developmental periods, might adversely effect the interpretation of results. Consequently, we employed nystatin-perforated patch-clamp recording techniques (Korn et al., 1991) to verify records obtained with standard whole-cell techniques. The perforation solution was made as follows: $10 \mathrm{mg}$ pluronic F-127 (Molecular Probes p-1572) was dissolved in $200 \mu$ d dimethyl sulfoxide (Sig$\mathrm{ma}$ ), and $10 \mathrm{mg}$ nystatin (Sigma) was dissolved in this solution to make the stock. Ten microliters of the stock were added to $5 \mathrm{ml}$ of filtered pipette solution to make the recording solution.

We could usually record whole-cell currents within 3-5 min after seal formation. Series resistance was increased in most instances to range from 20 to $40 \mathrm{M} \Omega$. Occasionally, cells developed an increased leakage current during prolonged recording, indicating that nystatin had penetrated the cell, but this was usually not a problem.

Motor nerve stimulation. Suction electrodes (tip diameter, $0.5-1 \mu \mathrm{m}$ ) were pulled on a Narishige patch-pipette puller and fire polished to achieve the desired configuration. A small segment $(0.5-1 \mu \mathrm{m})$ of the intact motor nerve was drawn into the pipette with gentle suction to form a tight seal. Stimulation was applied with a Farnell pulse-generating system and responses recorded from the patch-clamped myotube as described above. The shock artifact was decreased with the use of an isolated virtual ground.

L-Glutamate iontophoresis and glutamate receptor (gluR) mapping. A stock solution of $0.1 \mathrm{M} \mathrm{L-glutamate}$ (monosodium salt; Sigma) of $\mathrm{pH} 8$ was made fresh daily. Iontophoretic pipettes were pulled for specific requirements: high-resistance pipcttes $(100-200 \mathrm{M} \Omega)$ used for the glutamate receptor (gluR) mapping, and lower-resistance pipettes (50-75 $\mathrm{M} \Omega$ ) were used to analyze the glutamate response at the developing synapse. Glutamate was iontophoresed with short pulses $(<1 \mathrm{msec})$ of negative current ( $10 \mathrm{nA}$; Farnell pulse-generating system). Glutamate leakage between pulses was prevented with a small, positive backing current.

For the purposes of gluR mapping, the myotube surface was divided into a grid of $\sim 10 \times 10 \mu \mathrm{m}$ zones. Each zone was sampled in turn by advancing the iontophoretic pipette $10 \mu \mathrm{m}$ with a Leitz micromanipulator and delivering a pulse of negative current. Only the internal and lateral surfaces of the myotubes were available to glutamate iontophoresis in this dissection; gluR maps of the external myotube surface were extrapolated from these areas. [While calculating gluR statistics, we made the assumption that internal and external myotube surfaces are identical in terms of gluR numbers and distribution; we were unable to test this assumption directly.] Current responses in the voltage-clamped myotube were recorded continuously onto a digital audio tape recorder (Sony); myotube grid position was simultaneously recorded with an audio overlay. In most cases, individual gluR openings could be resolved and counted; the number of gluRs in massed gluR openings later in development was directly calculated by dividing the current amplitude by the amplitude of a single gluR opening.

A membrane-permeant fluorescent mitochondrial dye, rhodamine 123 (Sigma), was used to aid visualization of the developing nerve terminals in gluR mapping experiments (Yoshikami and Okun, 1984; Tse et al., 1991). The dissected embryo was stained in the dye $(2 \mu \mathrm{g} /$ $\mathrm{ml}$ in normal saline) for $5 \mathrm{~min}$ at $25^{\circ} \mathrm{C}$, and excess dye was removed with several washes of saline. The preparation was viewed with an epifluorescence attachment (Zeiss) and appropriate filters (blue excitation/green emission). In the time course of these experiments, the dye did not significantly alter any measurable electrophysiological properties of the preparation.

A commercially available arylamine spider toxin [Argiotoxin-636 pentahydrochloride (F.W. 819.13); Natural Sciences Inc. (lot 4)] which is a specific, noncompetitive, open-channel gluR block in both invertebrate and vertebrate preparations (for review, see Jackson and Usherwood, 1988), was used to characterize the gluR further and as a neuromuscular transmission block. We found that bath application of $10^{-5}$ M Argiotoxin-636 would completely block gluR openings within $5 \mathrm{~min}$ of application (filtered at $2 \mathrm{kHz}$ ). Iontophoretic application of L-glutamate (as above) elicited no response at junctional or extrajunctional sites, though we cannot preclude the possibility of very brief openings $(<100 \mu \mathrm{sec})$. This blockage was apparently irreversible, as no gluR openings were detected even after repeated washing $(>15 \mathrm{~min})$. The toxin was dissolved in MM3 medium $(10 \mu \mathrm{g} / \mathrm{ml})$ and either (1) applied directly to a cultured preparation or (2) pressure injected into an intact embryo with a beveled micropipette. For pressure injection, the embryo was slightly dehydrated ( $5 \mathrm{~min}$ in air) and toxin concentration and injection volume were adjusted to approximate $10^{5} \mathrm{M}$ toxin in the hemolymph.

Immunocytochemistry and scanning electron microscopy (SEM). Two antibodies were used in the course of this study: (1) anti-glutamate (antiglu; Arnel Products) and (2) anti-horseradish peroxidase (anti-HRP; Cappell), which recognizes a neuron-specific cell surface antigen in Drosophila (Jan and Jan, 1982). Fmbryos were dissected flat on polylysinecoated coverslips (as above) in normal saline to reveal the neuromusculature, fixed for $15 \mathrm{~min}$ in $4 \%$ paraformaldehyde, washed in phosphate-buffered saline plus $0.3 \%$ Triton X-100 (PBT), and blocked in $2 \%$ goat serum in PBT. The preparations were incubated in primary antibody (1:500 anti-HRP; 1:1000 anti-glu in PBT) for $1 \mathrm{hr}$ at RT with gentle agitation. For light microscopy, the preparations were again washed in PBT, incubated with the appropriate biotinylated secondary antibody (Vectastain; 1:200 dilution in PBT, agitation, $1 \mathrm{hr}$ at RT), washed, and incubated with a tertiary commercial avidin-peroxidase complex as directed ( $\mathrm{ABC}$ kit; Vectastain). The specimens were reacted with diaminobenzidine (DAB), cleared, and mounted for observation. For scanning electron microscopy (SEM), the procedure was as described above through incubation with the primary antibody. The preparations were then incubated in a secondary antibody coupled to a $1 \mathrm{~nm}$ gold particle (Biocell; 1:100 dilution in PBT, agitation, $1 \mathrm{hr}$ at RT), washed in PBT, and equilibrated in several changes of distilled water. The gold signal was silver-intensified with a commercially available kit (Biocell) while monitoring the progression of the reaction under a compound microscope. The preparations were postfixed overnight in 3\% glutaraldehyde, $0.5 \%$ paraformaldehyde. The preparations were dehydrated through an ethanol series, equilibrated with Freon, critical-point dried, and coated with 20-30 $\mathrm{nm}$ carbon. The specimens were viewed with a Jeol scanning electron microscope fitted with a back-scatter electron detector. The detector allowed the differentiation of the carbon and gold/silver signals.

Intracellular dye fills. After flat preparation (see above), myotubes and motor neurons were viewed with Nomarski optics and epifluorescence (as above) and iontophoretically injected with fluorescent dyes. Two dyes were used: (1) a $2 \%$ solution of I ucifer yellow-cadaverine-biotin (Molecular Probes) and (2) a solution of $2 \% N$-(2-aminoethyl)-biotinamide hydrochloride [Neurobiotin (FW 322.47); Vector Laboratories] and $2 \%$ Lucifer yellow. Lucifer yellow (and derivatives) was injected with small (nanoamps) hyperpolarizing current pulse under direct observation; to load Neurobiotin, the polarity of the current was reversed after confirming cell identity with the Lucifer yellow. Neurobiotin is small enough to traverse gap junctions, while Lucifer yellow-cadaverine-biotin is not. After injection, embryos were fixed for $1 \mathrm{hr}$ in $4 \%$ paraformaldehyde, washed with $\mathrm{PBT}$, and incubated with a commercial avidin-peroxidase complex (ABC Elite kit; Vectastain) for $30 \mathrm{~min}$. The specimens were then reacted with $\mathrm{DAB}$, cleared in xylene, and mounted to create a permanent preparation.

\section{Results}

\section{Morphogenesis of an identified NMJ}

Fach abdominal hemisegment (A2-A7) of the Drosophila embryo has 30 syncytial muscle fibers innervated by two peripheral nerves: the anterior intersegmental nerve (ISN), which innervates the dorsal musculature, and the posterior segmental nerve $(\mathrm{SN})$, which innervates the ventral muscles. The motor axons from these nerves form stereotyped neuromuscular synapses on muscles, which can be morphologically divided into two types (Fig. 1A): type I, characterized by short, localized terminal branches and large presynaptic release sites, or boutons; and type II, characterized by long, thin branches and numerous small boutons (Johansen et al., 1989a). In both types, the axon branches many times on the muscle surface and the finer branches (mostly type II) spread almost the entire muscle length, thus distributing synaptic contacts to the entire length of the muscle (Fig. 1 $A, B$ ). Most muscles receive both type I and lype II synaptic innervation, which attaches and arborizes on the muscle surface in a highly conserved, muscle-specific fashion (Johansen 

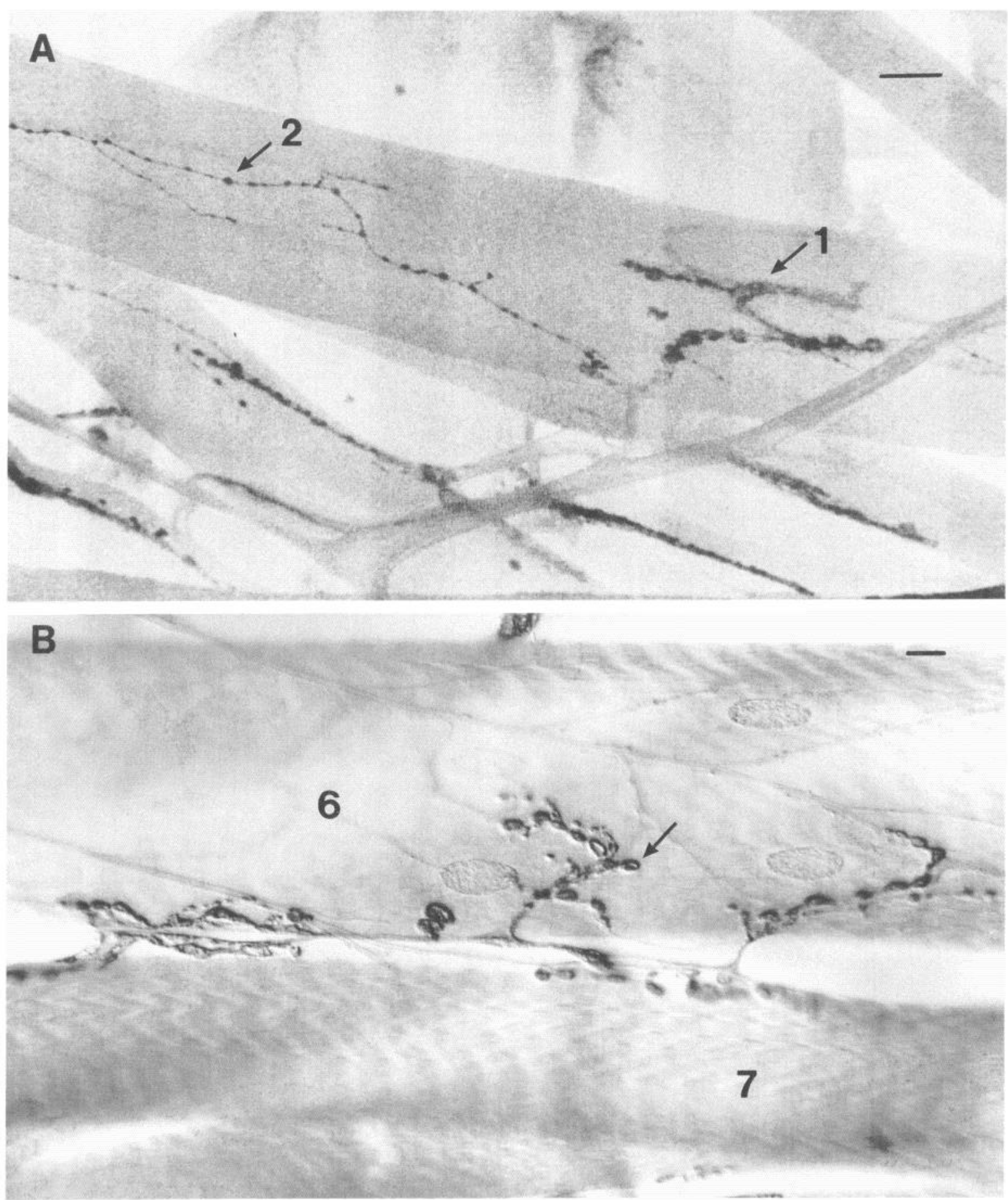

Figure 1. A, The morphology of the mature NMJs on muscle 4 in the third instar larva (A2). NMJs are widely distributed on the muscle surface, with little apparent postsynaptic modifications. NMJs can be divided into two morphological groups: short, thick, localized terminal arborization with large presynaptic boutons (varicosities) (I); and long, thin terminal branches with smaller boutons distributed evenly along their length (2). This preparation was stained with anti-HRP revealed with SEM techniques. $B$, The mature morphology of the NMJ on muscles 6 and 7 examined in this study, in the third instar larva (A2). This is the simplest NMJ in the larval abdomen, as it consists of only a single morphological type (type 1), represents the only synaptic input to these muscles, and releases only one known transmitter (L-glutamate). The muscles share a common motor neuron (RP3) that distributes boutons (arrow) longitudinally along the lateral cleft between the two muscles for approximately $50 \%$ of the muscle length, starting $20 \%$ of the length from the anterior end and extending posteriorly. This preparation was stained with anti-glu and examined with Nomarski optics. Scale bars, $10 \mu \mathrm{m}$.

et al., 1989a). Muscle innervation can be further subdivided according to neurotransmitter type: L-glutamate, octopamine, and proctolin have all been localized to subsets of the larval NMJs (Anderson et al., 1988). We have taken these factors into consideration in selecting the $\mathrm{NMJ}$ on the large, ventral, interior longitudinal muscle (muscle 6; Fig. 2) for developmental analysis. This is the simplest NMJ in the abdomen (Fig. $1 B$ ); it has only a single morphological type of junction (type I), a single 


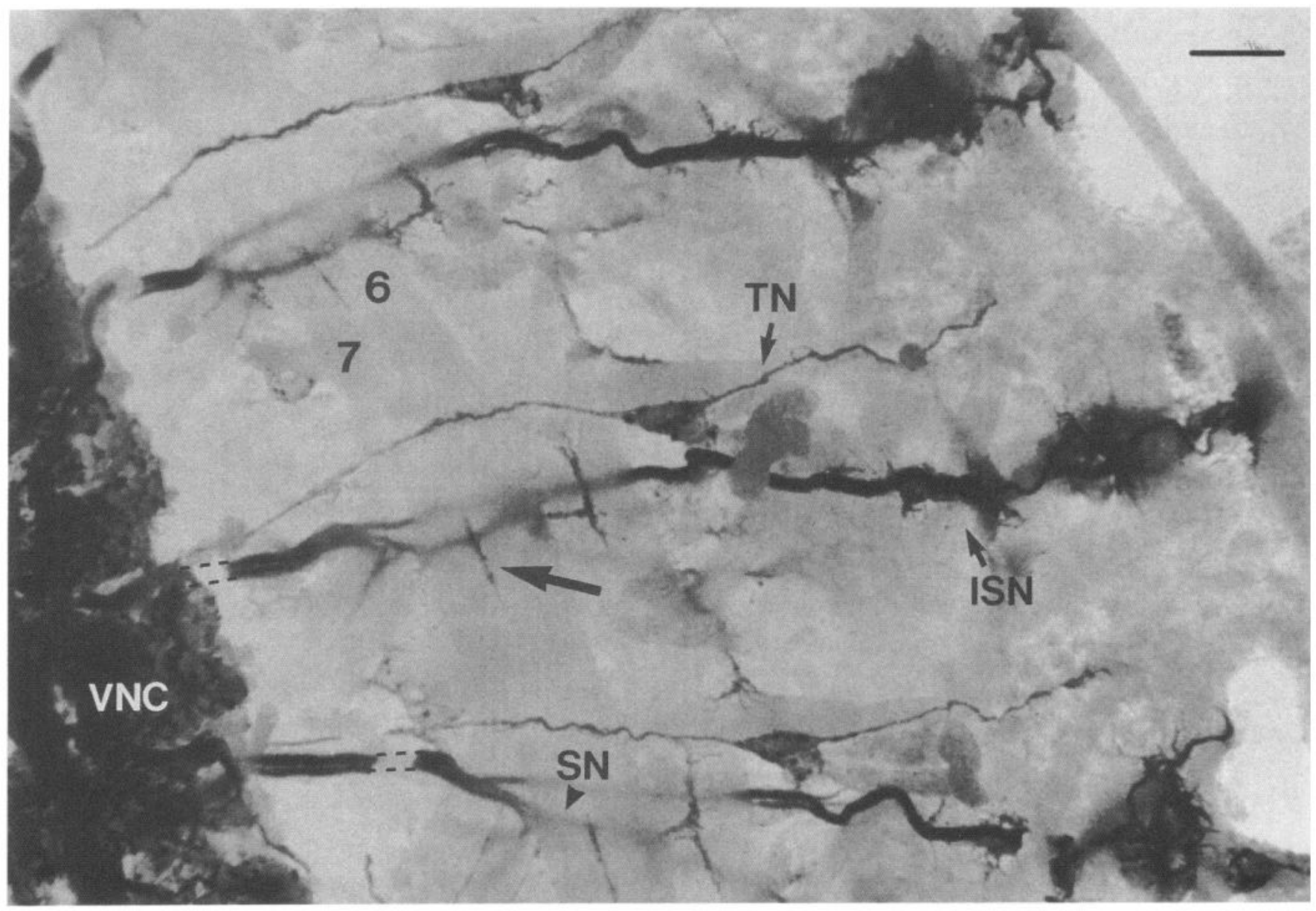

Figure 2. The morphology of the embryo neuromusculature in segments A2-A4 during the early stages of synaptogenesis (14 hr AEL). The embryo was dissected flat along the dorsal midline, stained with anti-HRP, and examined with SEM techniques; the ventral nerve cord (VNC) is to the left, the cut dorsal midline is to the right, and anterior is to the top. Muscles 6 and 7 are labeled. The NMJ examined in this study (arrow, A3) develops in the cleft between these two muscles. The ventral-projecting $\mathrm{SN}$, the dorsal ISN, and the transverse nerve (TN) are clearly revealed. Scale bar, $10 \mu \mathrm{m}$.

synaptic zone (along the medial-lateral margin with muscle 7), and a single known neurotransmitter (L-glutamate). Furthermore, it has been well characterized both physiologically and morphologically in the larva (Jan and Jan, 1976a,b; Johansen et al., 1989a) and examined morphologically in the embryo (Johansen et al., 1989b; Halpern et al., 1991; Sink and Whitington, 1991a,b), and its size and position make it the most amenable muscle for embryonic manipulation (Fig. 2). The complications are that it appears to be doubly innervated in the larva (Jan and Jan, 1976b) and shares innervation by at least one motor neuron (RP3; Sink and Whitington, 1991a,b) with adjacent muscle 7 (Fig. 1B).

Muscle 6 is innervated by one of the four branches of the ventral SN (SNb; Fig. 2). The $\mathrm{SN}$ is pioneered at 9.5-9.75 hr AEL, slightly delayed relative to the pioneering of the ISN (9$9.25 \mathrm{hr}$ AEL), immediately after completion of germ band retraction (8.75-9 hr AEL). Previous work (Halpern et al., 1991) has shown that RP3, the motor neuron that will eventually synapse on muscle 6 , begins axogenesis at $10 \mathrm{hr}$ AEL and sends its axon within the anterior commissure of the CNS to fascic-

Figure 3. The late development of RP3 and its innervation of muscles 6 and 7. Staged embryos were dissected flat along the dorsal midline, a dye injected into the RP3 cell body (see Materials and Methods), and the preparation examined with Nomarski optics; the ventral nerve cord is to the left, and anterior is to the top. $A$ and $B$, At $12 \mathrm{hr} \operatorname{AEL}(N=9$ cells $/ 5$ embryos), the RP3 growth cone is present in SNb but has not contacted muscles 6 and 7. A detail of an RP3 growth cone is shown in $B$. $C$ and $D$, At $12.75 \mathrm{hr}$ AEL $(N=12$ cells/7 embryos), the RP3 growth cone has contacted muscles 6 and 7 at a characteristic position in the anterior cleft between the two muscles. Prominent filopodia $(D)$ explore anterior and posterior in the cleft. $E$ and $F$, From 13 to $14 \mathrm{hr}$ AEL $(N=23$ cells/11 embryos; shown here at $13.5 \mathrm{hr}$ AEL), extensive filopodial exploration of the muscles occurs. The growth cone takes on a complex morphology with extensive arborization in the cleft between muscles 6 and 7 and apparently variable production of incorrect side branches $(F)$. These side branches often contact adjacent muscles, but we can detect no restriction to these contacts. $G$. Incorrect branches are quickly eliminated, and by $14 \mathrm{hr}$ AEL most motor neuron contacts are restricted to the cleft between muscles 6 and $7(N=14$ cells $/ 7$ embryos). By $14.5 \mathrm{hr}$ AEL $(N=10$ cells; not shown), all motor neuron contacts are restricted to the mature synaptic zone. $H$, The established NMJ at $16 \mathrm{hr}$ AEL $(N=11$ cells $/ 6$ embryos). The NMJ occupies the mature synaptic zone and has produced numerous varicosities, or boutons, characteristic of presynaptic transmitter release sites. Scale bars: $G, 10 \mu \mathrm{m}$ for $A, C, E$, and $G ; H, 3 \mu \mathrm{m}$ for $B, D, F$, and $H$. 

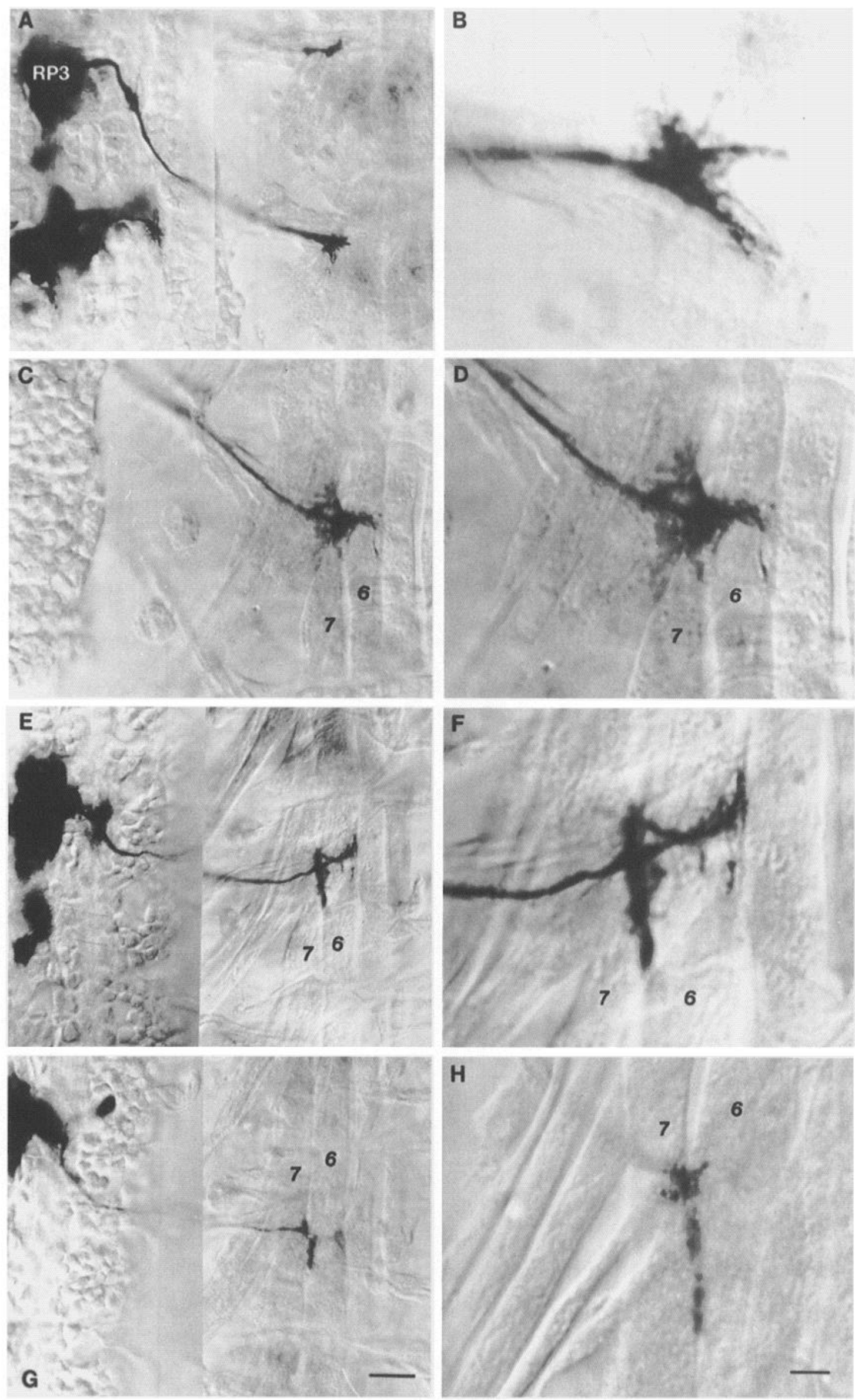

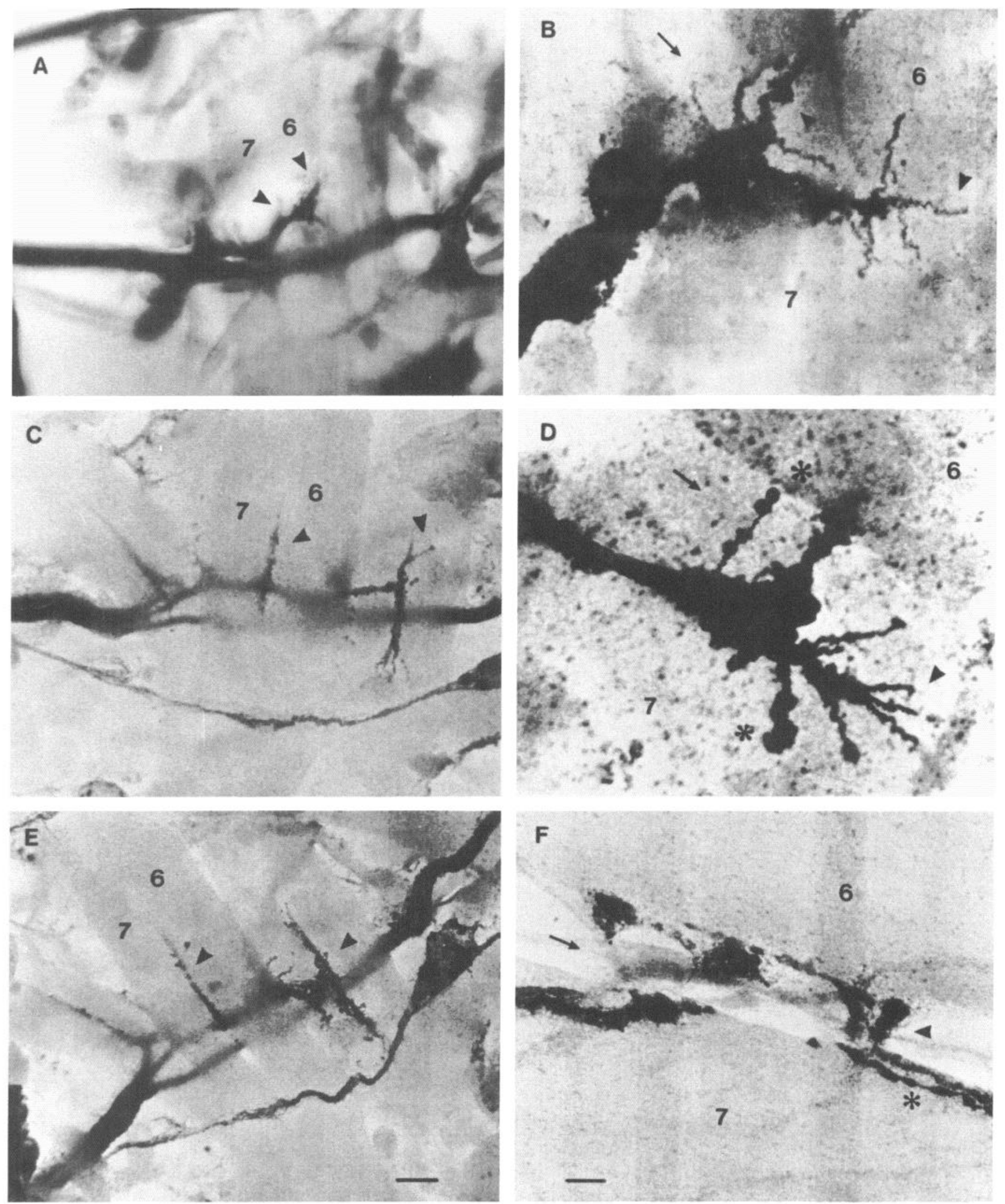

Figure 4. The morphological development of the NMJ on muscles 6 and 7. The preparations were stained with anti-HRP and examined with SEM techniques. Muscles 6 and 7 are labeled; posterior is to the top in $A, C$, and $E$ and to the right in $B, D$, and $F$. At least 10 embryos were examined at each $15 \mathrm{~min}$ interval from $12-16 \mathrm{hr}$ AEL and at $30 \mathrm{~min}$ intervals thereafter. $A$, At $12.5 \mathrm{hr}$ AEL, the SN is being actively pioneered; the upper and lower arrowheads indicate growth cones of $\mathrm{SNb}$, the lower of which will eventually synapse on muscle $6 / 7$. No contact with target muscles has yet occurred. $B$, At $13 \mathrm{hr}$ AEL, the initially contact between SNb and myotube 6/7. In early stages, growth cone filopodia (arrowheads) explore both anterior and posterior of the original contact point along the "synaptic cleft" (position indicated by arrow) between myotube $6 / 7$. $C$, 
ulate with other motor axons in the newly pioneered peripheral nerve. Over the next $2 \mathrm{hr}(10.5-12.5 \mathrm{hr}$ AEL), both the ISN and the SN extend over their appropriatc courses, with filopodial processes extending up to $10 \mu \mathrm{m}$ from the growth cone complex (Fig. 3A,B). The filopodial growth cones of RP3 and other motor axons of the SN project between two layers of the developing musculature, with developing myotubes 14 and 28 external and myotube 7 internal (Figs. 3, 4), navigating by means of littleunderstood mechanisms to the vicinity of their respective targets. Throughout this period of peripheral pathlinding, the mesoderm is differentiating into the mature muscle pattern (Bate, 1990). The mononucleate myoblasts complete fusion into multinucleated, syncytial myotubes (nascent muscle fibers), and the myotubes make insertions on the epidermis. By $12.5 \mathrm{hr} \mathrm{AEL}$, upon completion of dorsal epidermal closure, the mature muscle pattern has been achieved, the peripheral motor nerves have reached their mature extent in each hemisegment, and motor neuron growth cones are poised for synchronous exploration of the myotubes (Fig. 3C,D).

At 12.5-13 hr AEL, the RP3 motor neuron makes its initial contact on muscle 6 at a fixed anterior medial-lateral site in the cleft between muscles 6 and 7 (Figs. 3A-D, 4A), and its filopodia begin to explore the surface of both muscles. From the beginning, most of the filopodia are restricted to the cleft between muscles 6 and 7, part of which will form the mature synaptic site (Fig. 4B). Initially, prominent processes explore both anterior and posterior to the original contact site (Fig. $4 B$ ), but by 13.5 hr AEL the anterior processes have been retracted and all processes extend posterior of the original contact (Fig. 4C,D). Extensive processes also explore the immediate vicinity of the synaptic cleft during this period (Fig. $3 E, F$ ), often contacting adjacent muscles and forming substantial branches that differ both in length and thickness from the filopodia exploring the synaptic cleft of muscles 6 and 7 (Fig. $3 F$ ). The extent of this branching appears variable, as do the muscles these branches contact; multiple RP3 cells filled in the same embryo show highly variable terminal branching. It is not known if these branches actually synapse on adjacent muscles. These "incorrect branches" are transient and are eliminated by $14.5 \mathrm{hr}$ AEL (Fig. $3 G$ ). Concurrent with branching, from 13.5-14.5 hr AEL, filopodial processes explore posteriorly along the entire lateral margin of the synaptic cleft of muscles 6 and 7 , occasionally sending short processes laterally (i.e., ventral/dorsal of the cleft) for a few microns (Fig. $4 D$ ), which are soon retracted. During this period, it is clear that continuous motor neuron processes are spanning the cleft between muscles 6 and 7 and that the same motor neuron is arborizing on both muscles. By $14.5 \mathrm{hr}$ AEL, the posterior processes have retracted and are restricted to the medial-lateral two-thirds of the muscle, posterior to the original insertion site (Fig. $4 E$ ). Lateral processes have decreased in frequency and, by $14.5 \mathrm{hr}$ AEL, are no longer observed. Thus, by $14.5 \mathrm{hr}$ AEL, the motor neuron processes are restricted to the mature synaptic zone along the anterior-medial lateral margin.

The presynaptic terminals in the mature larva contain many varicose swellings, or boutons (Fig. $1 A, B$ ), which have been shown to contain concentrations of synaptic vesicles and are believed to represent transmitter release sites (Fig. $1 B$; Johansen et al., 1989a). Similar presynaptic "swellings" appear in the embryo soon after filopodial restriction is completed (14.5-15 hr AEL; Fig. 4D). Initially, these swellings are rare, only two or three per terminal, but over the next $2 \mathrm{hr}$ (15-17 hr AEL) the number of presynaptic boutons increases rapidly. There is a clear proximal-distal progression in the maturation of the terminal, with the proximal processes establishing boutons first, as more distal processes continue to explore the environment with long filopodial processes (Fig. 4D). By $17 \mathrm{hr}$ AEL, the motor neuron contact on muscle 6 (and muscle 7) has all the gross morphological characteristics of the mature NMJ (Figs. $3 H, 4 F$ ): (1) anterior medial-lateral neuron entry point; (2) arborizations, primarily posterior to the entry point, to two-thirds of the muscle length along the synaptic cleft; (3) a number of processes spanning the cleft between muscles 6 and 7; and (4) densely clustered boutons throughout the synaptic arborization. No significant morphological changes can be discerned at the SEM level in later embryonic stages ( $>18 \mathrm{hr}$ AEL).

\section{Development of double innervation}

Apparently contradictory evidence has suggested that muscle 6 is singly innervated (by RP3) in the embryo (Sink and Whitington, 1991a,b) and doubly innervated in the larva (Jan and Jan, 1976a), though it has been suggested (Halpern et al., 1991) that a morphological basis for double innervation may be seen in the anterior abdominal segments examined in this study. This discrepancy appears to arise because the muscle is originally singly innervated, but a second motor neuron innervates it in later embryonic stages. We carried out two physiological experiments to investigate double innervation in the embryo. (1) A suction electrode was used to stimulate the motor nerve repetitively with pulses of gradually increasing intensity, and excitatory junctional current (EJC) response was used to screen for multiple stimulus thresholds. ' Wwo thresholds were found in late embryonic stages ( $>16 \mathrm{hr}$ AEL) at which stimuli evoked EJCs with slightly different latencics (Fig. 5). Most frequently, the two responses summated, and only in a small subset of cases could temporally distinct peaks be observed. This is probably a reflection of the very short length of motor nerve $(<15 \mu \mathrm{m})$ separating the stimulation site from the nerve terminal. The lower-threshold EJC response was always smaller in amplitude, but the exact amplitude ratio varied with development time. (2) Repeated stimuli at threshold 1 were used to fatigue the EJC response completely, and the stimulus strength was jumped to threshold 2 to test for a second EJC. We observed that a slight increase in stimulus strength under these conditions resulted in

At $14 \mathrm{hr}$ AFI, extensive contact occurs between SNb and myotube 6/7 (arrowheads) along the cleft that will be the mature synaptic site. Just dorsal SNb growth cones are actively exploring myotubes 12 and 13. D, The first synaptic boutons (*) appear by 14.5 hr AEL. At this time, processes anterior to the original contact have been withdrawn and incorrect branches eliminated (see Fig. 3) and all processes are restricted posterior along the synaptic cleft (position indicated by arrow). Notice that boutons $\left({ }^{*}\right)$ are forming in proximal regions of the nerve contact while growth cone filopodia (arrowhead) persist distally and continue to explore the synaptic cleft. $E$, By $15 \mathrm{hr}$ AEL, nerve processes have arborized extensively on the myotube surfaces (arrowheads) and are restricted to the mature synaptic site in the cleft between myotubes 6 and 7 posterior to the nerve contact point. $F$, Al 18 hr AEL, the myotube $6 / 7$ synapse has gained its mature morphology including closely spaced synaptic boutons $(*)$ and numerous processes (arrowhead) spanning the cleft separating the myotubes (position indicated by arrow). Scale bars: $E, 5 \mu \mathrm{m}$ for $A, C$, and $E ; F$, $0.6 \mu \mathrm{m}$ for $B$ and $D$ and $1 \mu \mathrm{m}$ for $F$. 


\section{A. Threshold 1}

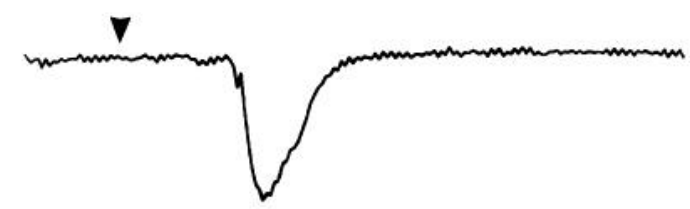

B. Threshold 2

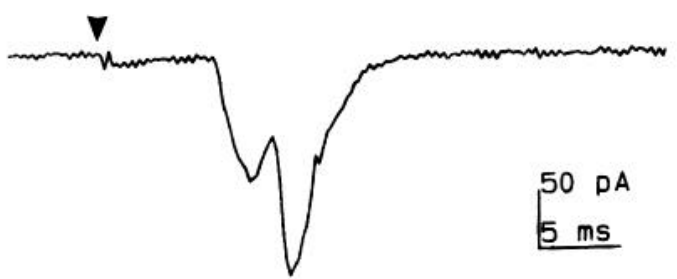

Figure 5. Evidence for double innervation of muscle 6 in the late embryo (17 hr AEL). Suction electrode stimulation was applied to the motor nerve (arrowheads), and the resulting EJCs were recorded in the myotube voltage clamped at $-60 \mathrm{mV}$. At a certain stimulation strength (threshold 1), an EJC response is recorded in the myotube $(A)$, which may increase slightly with stimulus strength. At a higher stimulus strength, a second threshold (threshold 2) is reached and a second EJC response $(B)$ recruited. Five of 17 embryos tested in this configuration positively demonstrated double innervation. This experiment was performed at decreased temperature $\left(14^{\circ} \mathrm{C}\right)$ to maximize the temporal separation of the two response peaks that are otherwise rarely resolved.

an immediate full-amplitude EJC consistent with the higherthreshold response (data not shown). Taking these data together, we conclude that muscle 6 is double innervated in the late embryo.

A single EJC response could be obtained at $13.5 \mathrm{hr}$ AEL, but we saw no evidence for double innervation prior to $15 \mathrm{hr}$ AEL and only routinely after $16 \mathrm{hr} \mathrm{AEL}$. We conclude that there are two periods of innervation: early innervation by RP3 (13.5$14.5 \mathrm{hr} \mathrm{AEL}$ ) and late innervation (15.5-16.5 $\mathrm{hr}$ AEL) by an unidentified motor neuron. There is little clear morphological evidence for the second innervation. Apparently, the synaptic sites of the two motor neurons are spatially overlapping and show no distinct morphological differences at the light or SEM levels. In particular, we saw no evidence that these motor neurons have type I and type II morphology distinctions; evidence for type II synaptic contacts on muscle 6 was rarely seen $(<5 \%)$. We stress that even in later stages ( $>18 \mathrm{hr}$ AEL), physiological evidence of double innervation was only clearly established in a subset $(25-30 \%)$ of muscles tested. Whether this is due to actual variability in the presence of the second motor neuron or mere technical difficulty is unclear.

\section{Development of neuromuscular activity}

By $13 \mathrm{hr}$ AEL, the mature muscle pattern is complete (Bate, 1990), but the muscles remain quiescent and are noncontractile when electrically stimulated or challenged with L-glutamate. By $13.5 \mathrm{hr} \mathrm{AEL}$, the myotube will contract when electrically stimulated, and endogenous contractions begin shortly thereafter (13.5-14 hr AEL). Muscle contraction in response to motor nerve stimulation occurs by $14.25 \mathrm{hr}$ AEL, indicating the presence of a functional NMJ. Muscle contraction can also be elic-
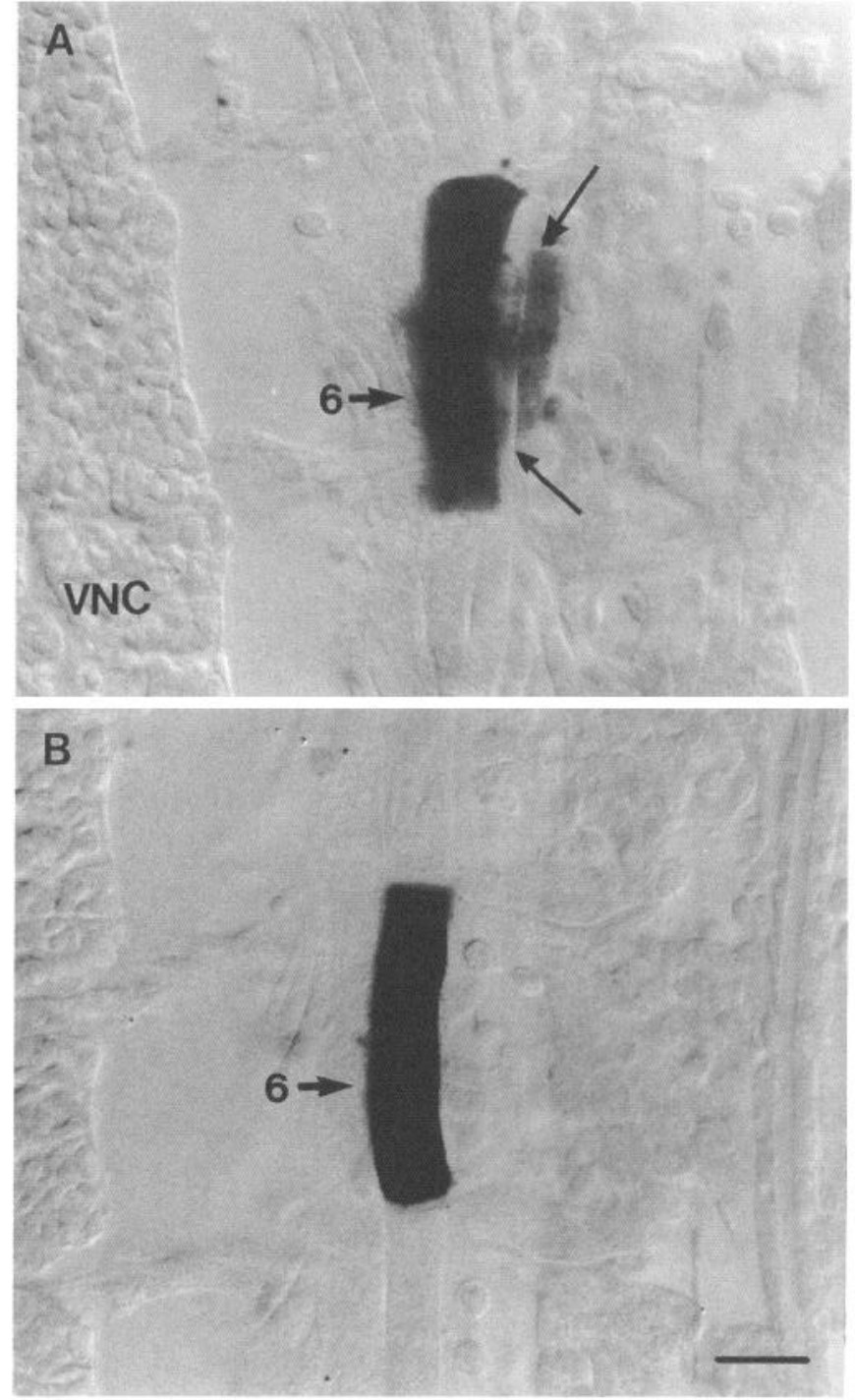

Figure 6. Muscles abruptly uncouple between 13 and $13.25 \mathrm{hr}$ AEL. Muscle 6 was injected with a dye (see Materials and Methods) for 5 min and the preparation examined with Nomarski optics; the ventral nerve cord $(V N C)$ is to the left, and anterior is to the top. $A$, At $13 \mathrm{hr}$ AEL ( $N=23$ of 27 cells/13 embryos), muscle 6 is coupled to several adjacent myotubes (arrows). Coupling usually extends to more external muscles (muscles 12-14, 26, 29), but the extent of coupling appears variable. Coupling between segments is occasionally observed $(N=4$ of 27). B, At $13.25 \mathrm{hr}$ AEL, muscle 6 retains the dye and few $(N=6$ of 43 cells $/ 20$ embryos) cells are dye coupled. The degree of coupling does not change dramatically over the next several hours $(N=2$ of 20 coupled at $16 \mathrm{hr}$ AEL). Scale bar, $10 \mu \mathrm{m}$.

ited by L-glutamate iontophoresis at the developing synapse at this time. Throughout this period, that is, $13.5 \mathrm{hr}$ onward, endogenous muscle contractions are increasing in frequency. Initially, only isolated myotubes contract, but by $14.5 \mathrm{hr} \mathrm{AEL}$, coordinated, peristaltic contractions of entire hemisegments begin. At $16 \mathrm{hr}$ AEL, there is an abrupt acceleration of peristaltic muscle contractions, and frequent muscle contractions, characteristic of larval locomotory movements, persist through to the end of embryogenesis. These movements are particularly frequent from 16-19 hr AEL but decline markedly in the hours prior to hatching ( $21 \mathrm{hr} \mathrm{AEL})$. Injection of a toxin (Argiotoxin$\left.636,10^{-5} \mathrm{M}\right)$ to block gluR activity stops most $(>80 \%)$ isolated 


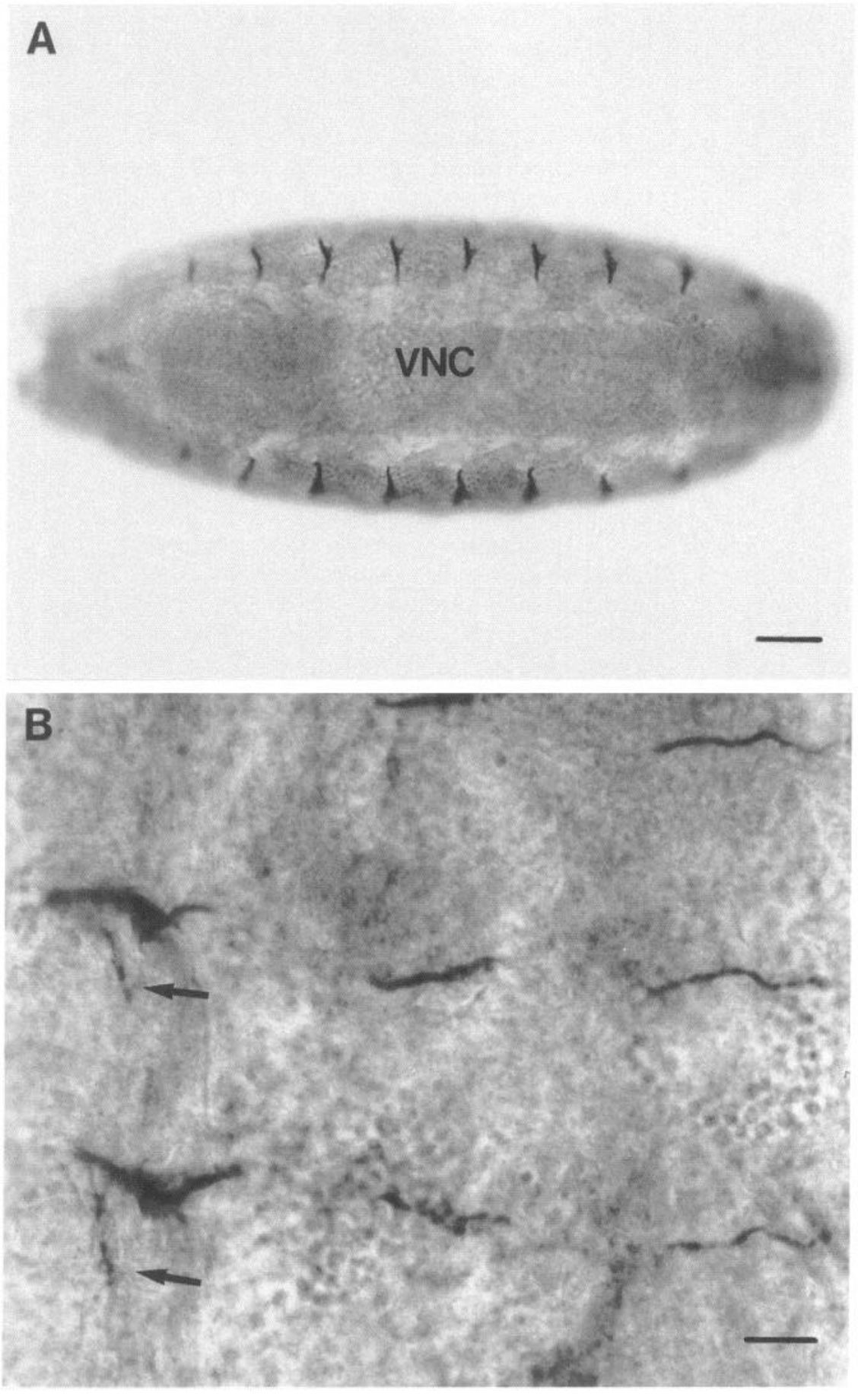

Figure 7. L-Glutamate immunoreactivity in the Drosophila embryo. A, At $12.5 \mathrm{hr}$ AEL, no immunoreactivity appears in the peripheral nerves $(N>100$; not shown). By $13 \mathrm{hr}$ AEL, the distal ends of the peripheral motor nerves have become intensely immunoreactive. $A$ shows a ventral view of an embryo at $13 \mathrm{hr}$ AEL. $V N C$, ventral nerve cord. Anterior is to the left. Nerves proximal to the ventral nerve cord do not stain; distal parts of both the ISN and SN (branching posteriorly) stain intensely. $B$, Lateral view of $13 \mathrm{hr}$ AEL embryo; ventral is to the left, and anterior is to the top. Arrows indicate the immunoreactive $\mathrm{SN}$ in $\mathrm{A} 2-\mathrm{A} 3$, which innervates muscles 6 and 7 . Notice that the dorsal ISN is also glutamate immunoreactive. Scale bars: $A, 50 \mu \mathrm{m} ; B, 15$ $\mu \mathrm{m}$. myotube contractions and all coordinated peristaltic movements, thus indicating that these movements are primarily glutamate mediated and probably result from neural activity at the developing NMJ.

\section{Cell coupling: muscle-muscle, muscle-neuron}

As in vertebrates (Blackshaw and Warner, 1976; Dennis et al., 1981), the myotubes of Drosophila are electrically and dye coupled for much of their early embryonic development (Johansen et al., 1989b), allowing the spread of depolarization laterally between fibers. During the early stages of synaptogenesis, the myotubes have attained the gross morphological character of the mature muscles (Bate, 1990) but remain coupled together as in earlier development (Fig. 6). Dye injection in muscle 6 at $13 \mathrm{hr}$ AEL reveals extensive coupling with adjacent muscles $(N=23$ of 27). Muscle 6 is most commonly coupled to more external muscles (muscles 12-14, 26, 29) at this development time, but the extent of coupling appears variable. Dye transfer is usually restricted within a segment, but occasional coupling to adjacent segments is observed ( $N=4$ of 27). Injection of current into muscle 6 can be detected in an adjacent muscle (muscle 13) during this period $(N=7$ of 10$)$. Between 13 and 13.25 hr AEL, the myotubes abruptly uncouple (Fig. 6). At 13.25 hr AEL, only 6 of 43 cells injected demonstrated detectable dye 


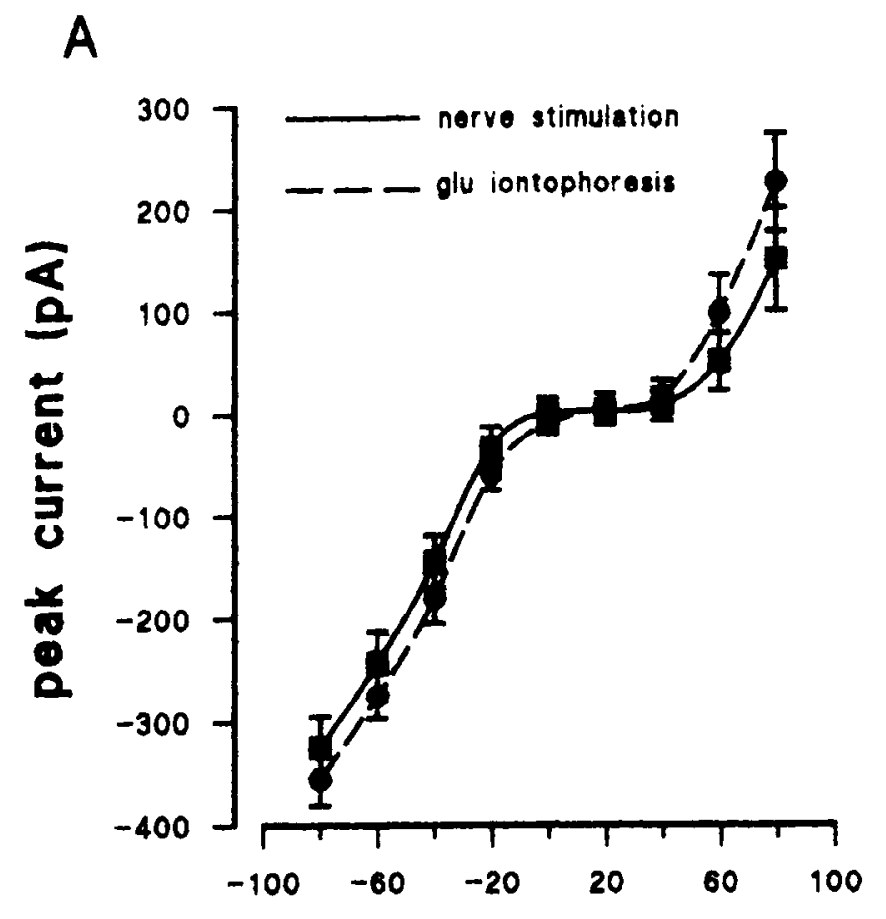

\section{command voltage $(\mathrm{mV})$}
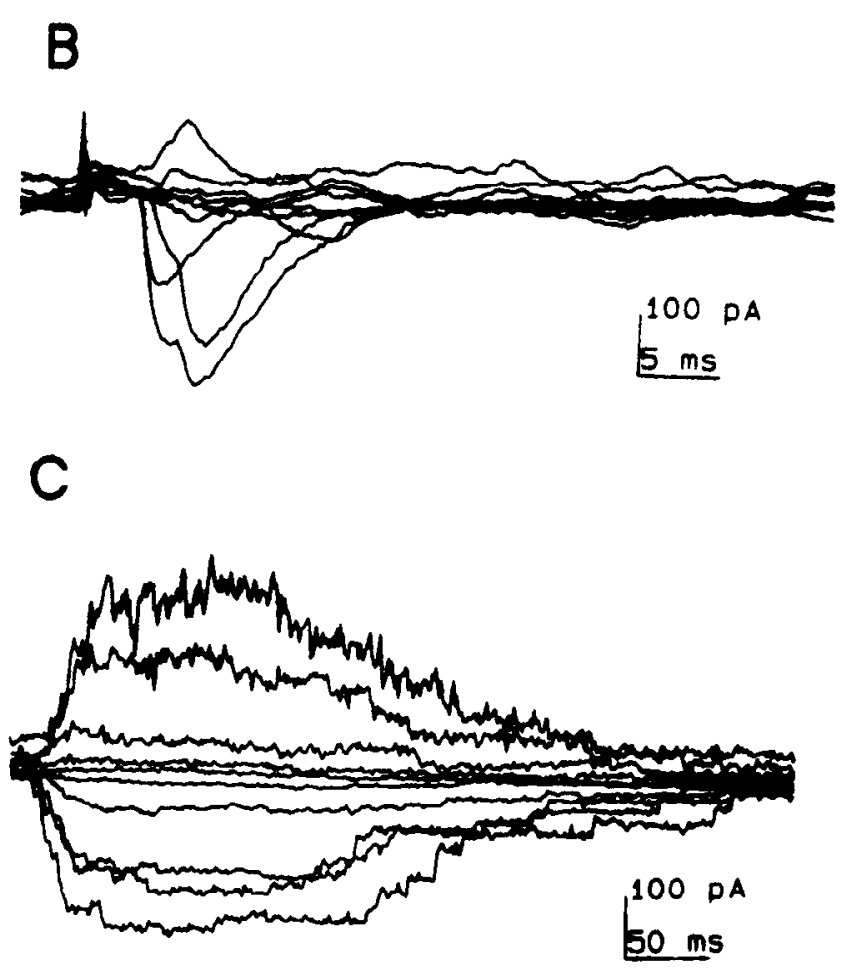

Figure 8. Excitatory currents at the developing NMJ of an embryonic myotube, $16 \mathrm{hr}$ AEL, elicited by suction electrode stimulation of the motor nerve and iontophoretic application of L-glutamate at the synaptic site. $A, I-V$ relationship of the synaptic current for both nerve stimulation and glutamate iontophoresis in the voltage-clamped myotube. Points are mean $\pm \mathrm{SD}(N=20) . B$ and $C$, Reversal potential of synaptic current for nerve stimulation $(B)$ and glutamate iontophoresis $(C)$ : myotube voltage clamped from -80 to $+80 \mathrm{mV}$ at $20 \mathrm{mV}$ intervals. transfer and electrical coupling with muscle 13 was not observed $(N=9)$. No dramatic change in coupling was observed in later development ( $N=2$ of 20 coupled at $16 \mathrm{hr}$ AEL). We note that this abrupt uncoupling closely correlates with the initial stages of synaptic development.

Studies in vertebrate cell culture systems (Allen and Warner, 1991) have also revealed a transient coupling between myotubes and their innervating motor neurons during the early stages of synaptogenesis, though such coupling has not been confirmed in vivo (Dennis et al., 1981). We tested for such coupling in Drosophila in two ways. (1) We investigated dye coupling by injecting Lucifer yellow into the myotube and analyzing the transfer of dye to the motor neuron, either directly with epifluorescence and/or using the Neurobiotin tracer to make a permanent preparation (see Materials and Methods). (2) We tested electrical coupling by blocking all chemical synaptic transmission, stimulating the motor nerve, and recording current responses in the voltage-clamped myotube. Chemical transmission was blocked by incubation in Argiotoxin-636 $(10 \mu \mathrm{g} / \mathrm{ml}$ MM3). Nerve stimulation under these conditions did not evoke muscle contraction $(N=12)$, and we observed neither spontaneous nor evoked currents during early synaptogenic stages (13.5-16 hr AEL). Furthermore, we detected no evidence of myotube-neuron dye transfer at any stage of early synaptic development (12.5-16 hr AEL; $N>5$ per 30 min interval). These negative findings do not exclude the possibility of rare transient coupling or coupling prior to the onset of synaptogenesis $(<12.5$ hr AEL).

\section{Maturation of transmitter expression}

During peripheral pathfinding (9-12.5 hr AEL), all motor neurons are glutamate negative in immunohistologically stained preparations. At $12.5-13 \mathrm{hr}$ AEL, high glutamate immunoreactivity is expressed in many motor neurons, including that innervating muscle 6/7, for the first time (Fig. 7). We were unable to determine whether glutamate expression commenced immediately prior to, during, or immediately following contact of the motor neuron with its target muscle (12.5-13 hr AEL). High glutamate immunoreactivity was localized to the distal end of motor axons and nerve endings on the muscle surface, but was not present in axon tracts closer to the CNS (Fig. 7). Glutamate immunoreactivity persists throughout embryogenesis with no obvious change in location or intensity.

\section{L-Glutamate as a neurotransmitter and properties of the embryonic gluR}

For a physiological investigation of synapse development, it was first necessary to define the elements of the embryonic NMJ. L-Glutamate and GABA have been reported independently as excitatory and inhibitory neurotransmitters, respectively, at the larval NMJ on muscle 6 (Jan and Jan, 1976a; Delgado et al., 1989). We found that $\mathrm{L}$-glutamate produced a large inward current when applied to the synaptic zone in late embryonic stages (Fig. 8); in contrast, similar application of GABA elicited no response at any embryonic stage. We found that the reversal potential for the glutamate-gated current was indistinguishable from the reversal potential for the synaptic current elicited by nerve stimulation (Fig. 8). In addition, the amplitude of the glutamate-gated current was the same as that of the nerve-evoked current in the late embryo (Fig. 8). This suggests that all gluRs can be saturated by both nerve stimulation and L-glutamate iontophoresis. Furthermore, all synaptic current in response to 
motor nerve stimulation could be irreversibly blocked with Argiotoxin-636 $\left(10^{-5} \mathrm{M}\right)$, which is known to block both vertebrate and invertebrate L-glutamate receptors (Jackson and Usherwood, 1988). These data, coupled with the high glutamate immunoreactivity of the presynaptic nerve terminals of the embryonic NMJ (Fig. 7) and the evidence from the mature larval NMJ (Jan and Jan, 1976a), strongly suggest that L-glutamate is the transmitter at embryonic NMJ on muscle 6.

Because of the high resistance (200-500 M $\Omega$ ) of the embryonic myotubes, the large conductance of the embryonic gluR (200 pS; Fig. 9A), and the low number of gluRs present in the early myotube, we found that individual gluR openings could be resolved easily in whole-cell patch-clamp configuration (Figs. $10 \mathrm{~B}$, 11). The characteristics of the gluRs were examined both with endogenous openings and openings elicited by iontophoretic application of L-glutamate. We found gluRs of only a single, large-conductance type $(200 \mathrm{pS})$, but with complex open times that suggest at least three open states (means of $0.23,2.1$, and $11.8 \mathrm{msec}$; Fig. $9 B$ ). The majority of openings $(<90 \%)$ at all development stages were brief $(<1 \mathrm{msec})$, with long openings $(>10 \mathrm{msec})$ relatively rare $(<5 \%, 15 \mathrm{hr}$ AEL). The frequency of long openings ( $>10 \mathrm{msec}$ ) decreased even further with development time $(<1 \%, 21 \mathrm{hr} \mathrm{AEL})$. Whether these data reflect the presence of a heterogeneous population of gluRs or a single type with multiple open states cannot be resolved in whole-cell recording. However, preliminary data with cell-attached patchclamping of single gluRs suggest a single-channel type with multiple open states depending on L-glutamate concentration (data not shown). In these studies, the frequency of long openings (>10 msec) increased with glutamate concentration, suggesting that there may be higher glutamate concentrations in the synaptic cleft in early development, rather than that there are multiple gluR types or that there is a decrease in the frequency of a long-open-time embryonic gluR.

Openings of gluRs longer than $14 \mathrm{msec}$ were always interrupted by brief closures, characteristic of bursting behavior. Unlike other insect gluRs (Dudel et al., 1988), we found no evidence for rapid inactivation (desensitization) even after prolonged glutamate application (Fig. 10B). The typical bursting response was for the gluR channel to remain open for the duration of the exposure with only brief closures $(<0.2 \mathrm{msec})$.

\section{Clustering of gluRs at the developing synapse}

The response of localized domains of embryonic myotube membrane to iontophoretically applied L-glutamate was examined by observing currents under whole-cell voltage-clamp conditions. During early myogenesis $(<13 \mathrm{hr}$ AEL), we detected no functional gluRs in the myotube membrane $(N=15)$. Functional gluRs are first present in the myotube membrane at 13.25-13.5 hr AEL (Figs. 10, 11), immediately after uncoupling of the myotubes ( $<15 \mathrm{~min}$ ), and following the initial nerve-muscle contact (12.5-13 hr AEL; Figs. 3C,D;4A,B). Initially, the gluRs are homogeneously distributed over the exposed myotube surface and show no significant clustering either at the future synaptic zone or elsewhere (Fig. 10). However, significant clustering of gluRs at the developing synaptic zone begins by $13.75-14 \mathrm{hr}$ AEL, together with a concurrent decrease in extrajunctional receptors (Fig. 10). The progression of early gluR clustering follows a sigmoidal time course, initially slow but increasing rapidly with time. As gluR clustering progresses, the number of extrajunctional receptors decreases, but the total number of gluRs per myotube remains constant after reaching an initial peak at

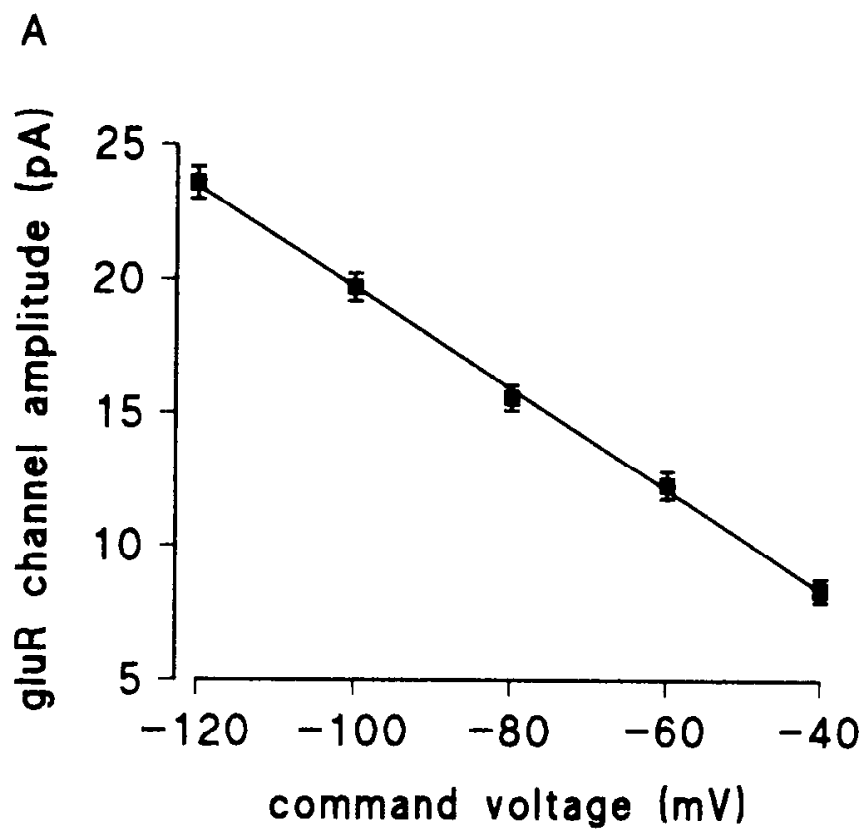

B

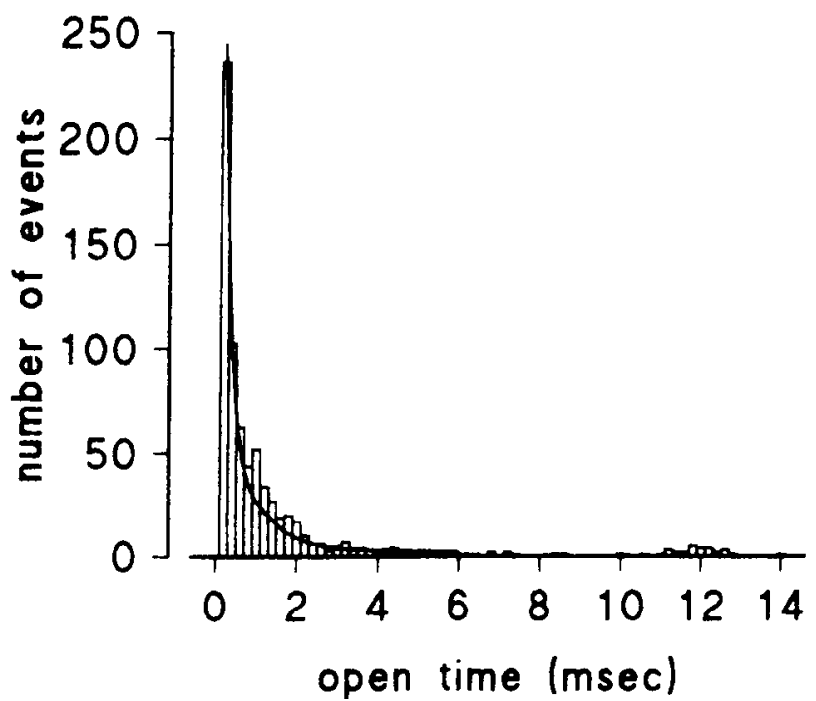

Figure 9. Single-channel characteristics of the gluR at the developing NMJ of an embryonic myotube, $16 \mathrm{hr}$ AEL, measured in whole-cell patch clamp configuration. $A, I-V$ relationship of the gluR current in the voltage-clamped myotube. Measurements at and above $-20 \mathrm{mV}$ cannot be accurately obtained due to the activation of voltage-gated channels and the decreasing amplitude of the gluR current. Points are mean $\pm \mathrm{SD}(N>100) . B$, Open times for the gluR channel measured in whole-cell configuration. The vast majority of openings $(<95 \%)$ can be fit to a double exponential equation, where $T_{f}=0.23 \mathrm{msec}$ and $T_{s}$ $=2.1 \mathrm{msec}$. Longer openings (mean $=11.8 \mathrm{msec}$ ) are also observed, but their rare occurrence make them difficult to fit. Openings longer than 14 msec are never observed, unless interrupted by brief closures characteristic of bursting activity. Examples of single-channel openings can be seen in Figures $10 \mathrm{~B}, 11$, and $15 \mathrm{~A}$.

$14 \mathrm{hr}$ AEL (Fig. 10C). By 15.5-16 hr AEL, all gluRs are restricted to the synaptic zone of the myotube surface (Fig. 10); no extrajunctional gluRs are detected at this stage or in later embryonic stages.

We wished to know whether gluR clustering at the developing synaptic contacts occurs through lateral movement of previously unlocalized gluRs (gluR aggregation), the selective expression 
A

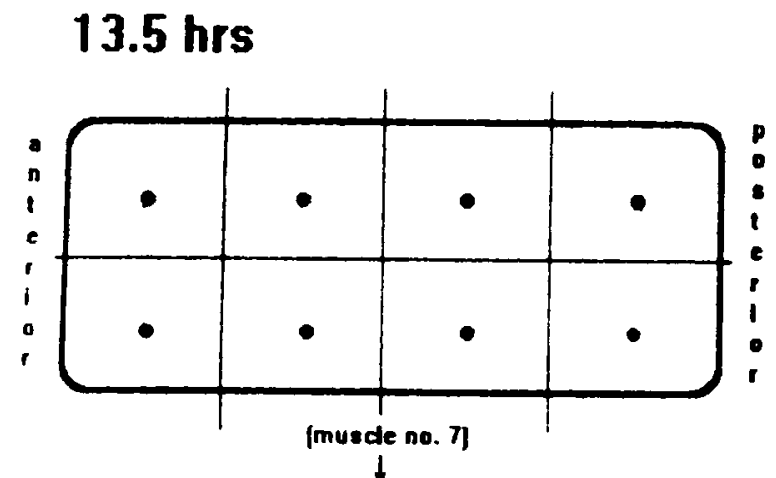

$14 \mathrm{hrs}$

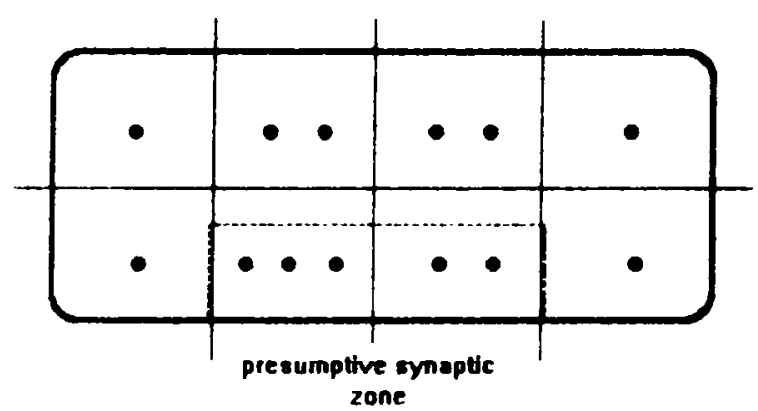

$14.5 \mathrm{hrs}$

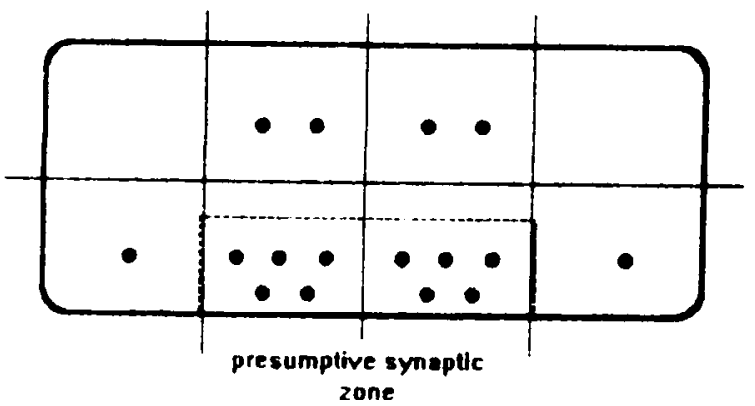

15 hrs

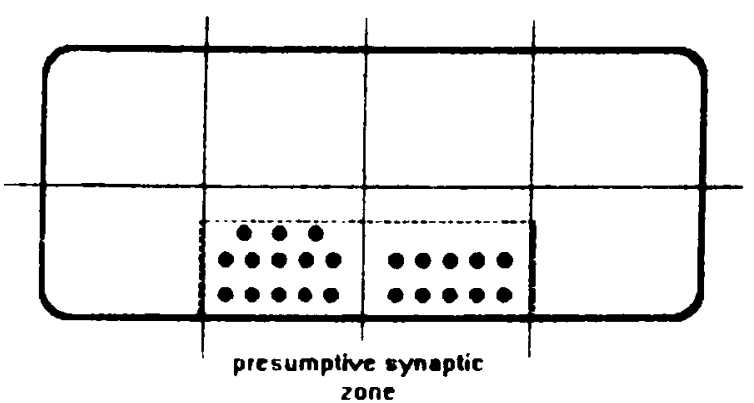

B
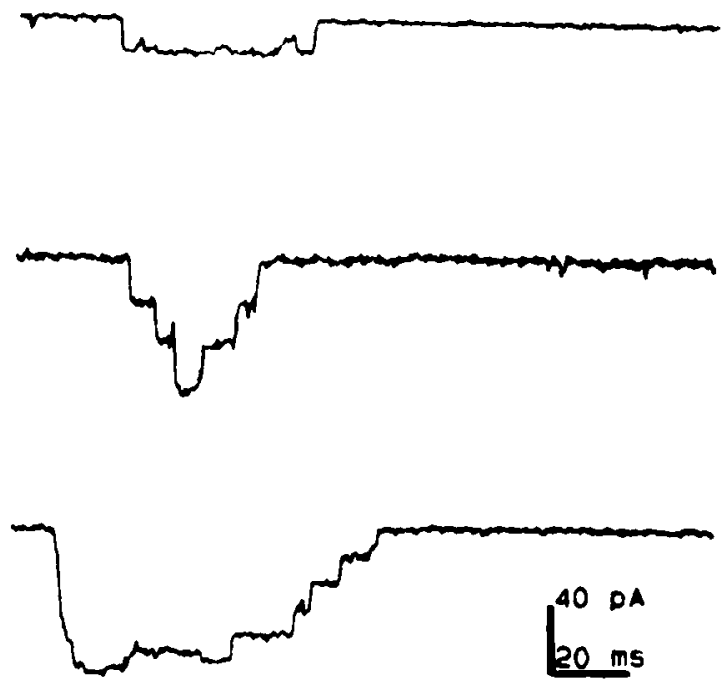

Q

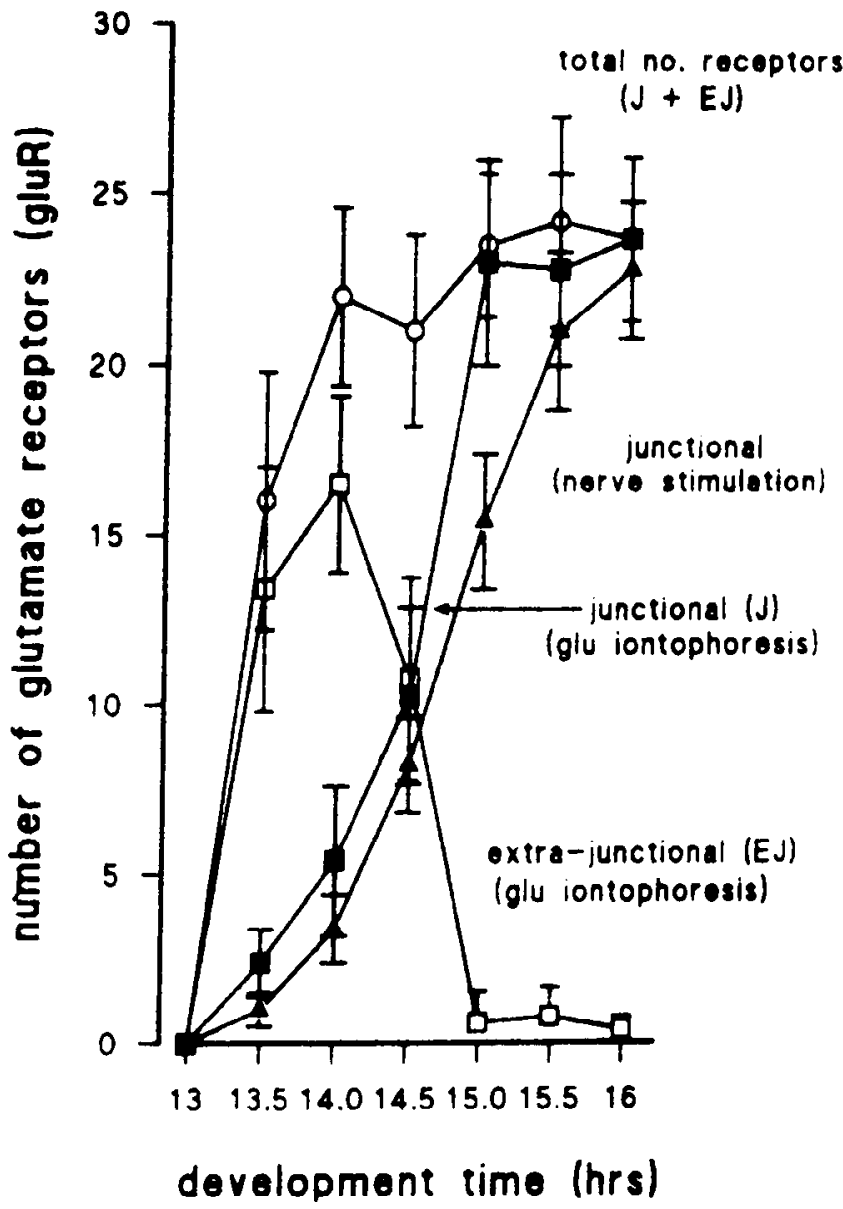

Figure 10. Clustering of L-glutamate receptors in the myotube synaptic zone during formation of the NMJ on muscle 6 . The distribution of gluRs was mapped by localized L-glutamate iontophoresis over the myotube surface while in whole-cell voltage-clamp (see Materials and Methods). $A$, Schematic diagram of the time course of gluR clustering from 13.5 to $15 \mathrm{hr}$ AEL. Each circle represents a single gluR, the solid grid outlines the iontophoretically sampled area, and the dotted box delineates the mature synaptic contact area. Anterior, posterior, and the position of the adjacent ventral myotube (myotube 7) are indicated. The interior surface of the developing myotube is exposed. $B$, Three examples of gluR current responses 
of new gluRs at the synaptic sites (gluR synthesis) and concurrent loss of extrajunctional gluRs, or a combination of these two processes. To test this, we blocked the function of gluRs at 14 hr AEL, at the peak of total gluR numbers but early in the gluR clustering process (Fig. $10 \mathrm{C}$ ), by incubating for $10 \mathrm{~min}$ in Argiotoxin-636 (10-5 $\mathbf{M}$; see Materials and Methods). The embryo was then washed free of toxin and cultured for $1 \mathrm{hr}(\sim 15 \mathrm{hr}$ AEL), and L-glutamate was iontophoresed at the synaptic contacts to test for the presence of functional gluRs. Since Argiotoxin-636 blocks gluR openings irreversibly (Jackson and Usherwood, 1988), all functional receptors would have been newly synthesized since the toxin incubation. Using this experimental design, we found very few functional gluRs at the synaptic contacts (mean $=4 \pm 2 ; N=8$ ), relative to the number of gluR clustered during this interval (14-15 hr AEL) in the unblocked control (mean $=17 \pm 5 ; N=10$ ). This result, coupled with the fact that the total number of gluks is highly conserved during the normal clustering process (Fig. $10 \mathrm{C}$ ), suggests that clustering initially occurs predominantly through aggregation of precxisting gluRs and that there is little, if any, site-directed synthesis of new gluRs.

Expression of gluRs occurs in two phases. First, there is an early homogeneous expression of a small number of gluRs $(<25)$ beginning at $13.25 \mathrm{hr}$ AEL and complete by $14 \mathrm{hr}$ AEL (Fig. $10 C)$. Second, there is a late expression of a greater number of gluRs ( $>50$ ) starting at 16.5-17 hr AEL and continuing through the end of embryogenesis (Figs. 11, 12). Between these two phases (14-16.5 hr AEL), there is no significant increase in the total number of gluRs on muscle 6 (Fig. 12). Unlike the initial expression phase, the second phase seems to be site directed to the synaptic zone; no extrajunctional gluRs are detected. Alternatively, insertion in the membrane during both phases may be random, but localization of receptors late in development may be too rapid to be detected.

We could detect no differences in whole-cell configuration in the properties of gluRs in the two populations of a kind that would suggest that the early population is composed of specialized, embryonic gluRs. However, a comprehensive singlechannel analysis was not undertaken, and it remains possible that the two populations may have other distinctive characteristics, which correlate with their separate times of origin.

\section{Development of synaptic currents}

The maturation of synaptic currents was traced both by iontophoresis of glutamate at the developing synaptic site and by stimulating the developing nerve. An EJC was first observed soon after uncoupling of the myotubes: at $13.25 \mathrm{hr}$ AEL with iontophoresis, and at $13.5 \mathrm{hr}$ AEL with nerve stimulation (Fig. 11). In both cases, the earliest EJCs consisted of single, temporally distinct gluR openings; multiple openings were rare and of low amplitude (less than three gluRs; Figs. 11, 12). Simultaneous multiple-gluR-opening EJCs developed soon afterward, initially (13.5-14.5 hr AEL) as low-amplitude events consisting of a few gluRs $(<10$; Fig. 11). A peak amplitude was reached

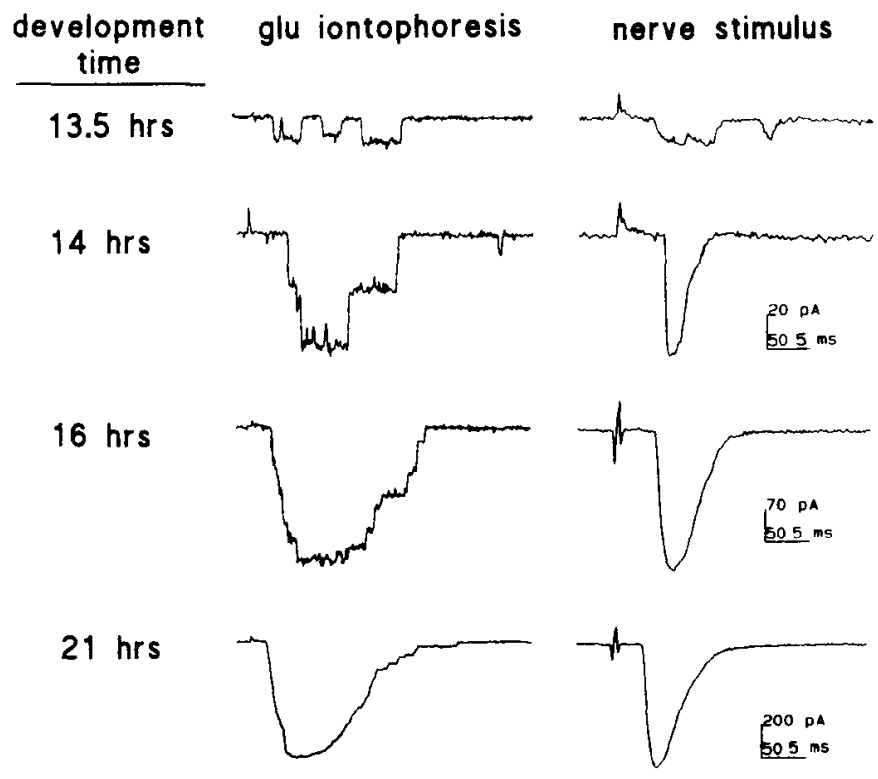

Figure 11. Maturation of the synaptic current at the developing NMJ on myotube 6 measured in response to L-glutamate iontophoresis and suction electrode stimulation of the embryonic motor ncrve. Shock artifacts indicate stimulation time.

by $15-15.5 \mathrm{hr}$ AEL, with a plateau showing no significant increase in EJC amplitude that lasted until 16.5-17 hr AEL. In later stages ( $>17 \mathrm{hr}$ AEL; Figs. 11, 12), there was a gradual increase in EJC amplitude that continued through embryogenesis. The time course of the EJCs did not change significantly during synaptogenesis; the falling phase of all EJCs decayed exponentially (Fig. 11).

It is striking that there was no significant difference in the amplitude of the synaptic currents elicited by glutamate iontophoresis at the developing $\mathrm{NMJ}$ and nerve stimulation during development (Figs. 11, 12). We conclude that all gluRs at the developing synaptic site are available to transmitter released from the nerve terminals. In contrast, there is a large difference in the time course of the synaptic currents elicited by the two methods (Fig. 11), a discrepancy that is probably attributable to technical difficulties. The duration of the developing synaptic current $(<10 \mathrm{msec}$ with nerve stimulation) did not change significantly during development and was similar to the time course of endogenous EJCs.

\section{Initiation of endogenous synaptic communication (13.25-14.5 hr $A E L$ )}

Endogenous currents are present from the first appearance of gluRs at $13.25 \mathrm{hr}$ AEL. This temporal correlation and the fact that these spontaneous currents have the same amplitude and duration distribution as the glutamate-gated channel currents lead us to conclude that these spontaneous currents represent gluR openings. The amplitudes of the miniature currents are

to localized $\mathrm{L}$-glutamate iontophoresis in embryonic myotubes voltage clamped at $-80 \mathrm{mV}$ during gluR mapping experiments. The traces show a single gluR opening, three gluR openings, and six gluR openings, respectively. The number of gluRs at each position was calculated from the peak response in each case. $C$, The time course of gluR clustering at the developing NMJ. The number of extrajunctional $(E J)$ gluRs was obtained by adding the responses from localized iontophoretic applications in all extrajunctional zones at each developmental period. The number of junctional $(J)$ receptors was obtained in a similar fashion from iontophoretic responses at the synaptic zone and, more precisely, by measuring the response to suction electrode stimulation of the motor nerve. The total number of gluRs on the myotube was obtained by adding junctional and extrajunctional receptors. Points are mean $\pm \mathrm{SD}(N=10$ embryos $)$. 
Figure 12. A summary of development of the NMJ using all the configurations discussed in the present study: suction electrode stimulation of the developing motor nerve (evoked EJC), L-glutamate iontophoresis at the developing synapse (junctional) and with addition of extrajunctional locations (whole cell), and measurement of endogenous synaptic currents. In all cases, the EJC amplitude was measured in whole-cell patch-clamp configuration in a myotube voltage clamped at -60 $\mathrm{mV}$. Three stages of synaptogenesis are described: (1) appearance of functional gluRs homogeneously distributed in the myotube membrane (13.25-14 hr AEL), (2) clustering of gluRs at the synaptic contact site (13.5-15.5 hr AEL), and (3) a second period of gluR synthesis (17 hr AEL through end of embryogenesis). Development time is measured in hours AEL; all points represent the mean \pm $\mathrm{SD}(N=10$ embryos $)$

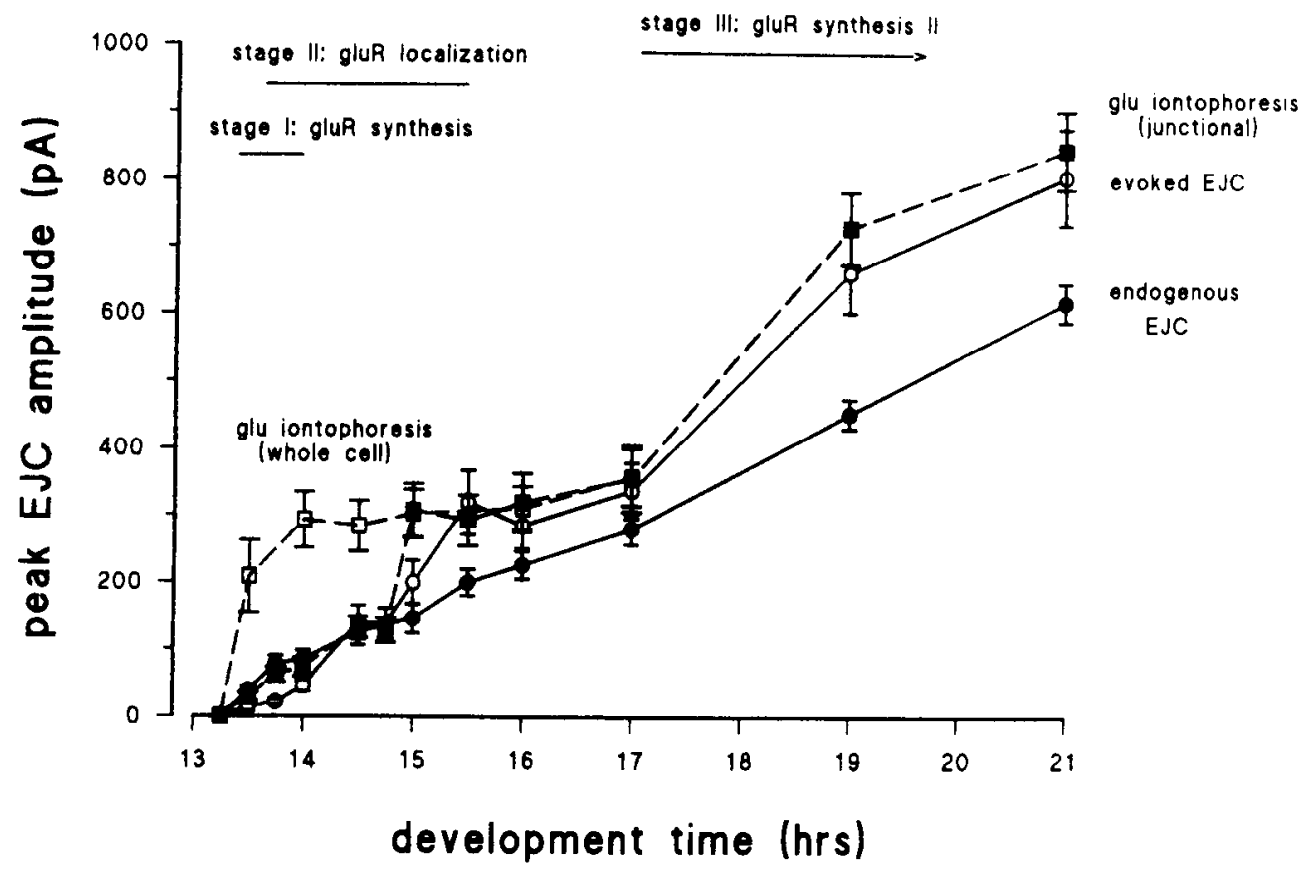

distributed in a normal multimodal fashion during synaptogencsis (Fig. 13). The lowest distribution had a mean amplitude corresponding to the current amplitude of a single gluR opening, and larger currents had peak distributions at regular intervals equal to the amplitude of a single gluR opening (Figs. 9, 13). We conclude that endogenous currents are based on a quantal unit equal to a single gluR opening.

Initially (13.25-13.5 hr AEL), endogenous currents show a monomodal distribution of single openings, though occasional double openings are observed, such as might be expected of spontaneous events (Fig. 13). However, simultaneous multiple gluR openings soon develop (13.5-13.75 hr AEL; Fig. 13), which must be in response to an external stimulus, presumably glutamate released from the exploring motor nerve growth cones. Larger, recognizable EJCs (>50 pA) are present by $14 \mathrm{hr}$ AEL, and the amplitude of these events continues to increase gradually throughout the later stages of synaptogenesis. The amplitude of the EJCs is observed to increase in quantal units equal to the amplitude of a single gluR opening (Fig. 13). At all stages, endogenous single gluR openings are the most common event.

Three types of endogenous currents are present during early (13.5-14.5 hr AEL) synaptogenesis (Figs. 14A, 15A): (1) "miniature" EJCs composed of only single or double gluR openings, which can either be isolated openings (all $<14 \mathrm{msec}$; $90 \%<1$ msec) or prolonged bursting openings (up to seconds) separated by brief closures ( $<0.2 \mathrm{msec}$ ); (2) maturing EJCs of low amplitude ( $<10$ gluR openings) and brief duration $(<10 \mathrm{msec})$; or (3) long-time-course EJCs usually lasting up to several seconds, but occasionally persisting for tens of seconds. The amplitude of this long-lasting EJC increases concurrently with the maximal short-duration EJC and seems to represent the long-term simultaneous opening of all the gluRs available to transmitter at the developing synapse. Both the long and short EJCs can be blocked with Argiotoxin-636 $\left(10^{-5} \mathrm{M}\right)$. Likewise, both the short and long EJCs can be blocked with standard synaptic blocks (decreased external $\mathrm{Ca}^{2+}$ and/or augmented external $\mathrm{Mg}^{2+}$ ) but miniature currents (single/double gluR openings) continue at a decreased frequency. This leakage of miniature currents is probably attributably to incomplete transmission blockage and/or release of $\mathrm{L}$-glutamate from adjacent tissues.

\section{Early synaptic potentials $(13.25-14.5 \mathrm{hr} A E L)$}

The basic units of the excitatory junction potential (EJP) are miniature potentials $(2-4 \mathrm{mV})$, present from the earliest stages of endogenous gluR openings at $13.25 \mathrm{hr}$ AEL (Figs. 14D, 15D). There is no evidence of inhibitory potentials (Fig. 14D). As in the vertebrate NMJ, larger synaptic potentials are a summation of these miniature "quanta." However, unlike in vertebrates, these quanta are not the response of many receptors to the release of a synaptic vesicle, but rather the consequence of opening a single gluR. Due to the high conductance of a gluR (Fig. 9), and the high input resistance of the embryonic myotube, the opening of a single gluR results in a large miniature potential $(2.8 \pm 0.6 \mathrm{mV}$; Fig. $15 \mathrm{D})$ similar in amplitude to miniature end-plate potentials at the vertebrate NMJ. This surprising fact explains why relatively large FJPs (20-30 mV; Figs. 14D, 15D) are already present at the earliest stages of synaptogenesis, when few gluRs $(<10)$ are clustered at the developing synaptic site (Fig. 10). It also explains how a functional NMJ (i.e., muscle contraction in response to nerve stimulation) is present very early in development (14.25 hr AEL). Even the 10-15 gluRs present at the developing synapse are sufficient to reach the threshold of the inward calcium current (see following companion article, Broadie and Bate, 1992) and so elicit a muscle contraction.

As predicted from the synaptic currents, most of the EJPS during the initial stages of synaptogenesis are miniature events $(<10 \mathrm{mV})$. By far the most common is the single-gluR-opening event $(2-4 \mathrm{mV})$; the rarer, larger events represent multiples of this basic unit (Fig. 14D). The largest events during early synaptogenesis $(<14 \mathrm{hr}$ AEL) depolarize the myotube by $25-35$ $\mathrm{mV}$ but fail to overshoot zero potential (Fig. 14D). Overshooting 
EJPs are first observed at the time a functional synapse is formed (14.25 hr AEL) and become increasingly common with development time. All EJPs during these early stages of synapse formation have very prolonged time courses relative to the mature potential changes; miniature potentials last $0.25-0.5 \mathrm{sec}$ and macro EJPs for shorter $(1-2 \mathrm{sec}$ ) and longer (up to $30 \mathrm{sec}$ ) periods (Figs. 14D, 15D). We conclude that the shorter EJPs represent the brief EJCs and the longer EJPs result from the prolonged synaptic currents.

\section{Development of endogenous synaptic communication (14.5-18} hr AEL)

By $14.5 \mathrm{hr}$ AEL, a functional NMJ has been established and the earliest stage of synapse formation is thus completed. No abrupt change in synaptic currents occurs during or after the establishment of this functional synapse. The amplitude of the EJCs continues to increase gradually (Figs. 12, 13). EJC amplitudes range from single gluR currents to the increasing maximum EJC amplitude; single openings remain the most common event, but macro EJC amplitudes are randomly distributed (Fig. $14 B$ ). The EJC duration ( $<10 \mathrm{msec}$ ) remains unaltered. Very long-duration EJCs (tens of seconds) persist but with ever declining frequency (Figs. 14B, 15B). As earlier, the amplitudes of the long- and short-duration EJCs remain comparable (Fig. $15 \mathrm{~B})$. The overall frequency of synaptic currents is decreased from carlicr stages, but the frequency of larger currents $(>10$ gluR openings) is increased and continues to increase in later developmental stages (Fig. 14B).

Unlike the EJCs, the characteristics of the EJPs change dramatically in the maturing synapse. Initially (14.5-15 hr AEL), the EJPs maintain the long-time-course ( $>1 \mathrm{sec})$, nonspiking character of earlier stages (Fig. 14D), only increasing gradually in amplitude. In later stages ( $>15 \mathrm{hr}$ AEL), a short-time-course $(<0.2 \mathrm{sec})$, spiking EJP develops (Figs. 14E, 15E). Initially, the long-time-course EJPs predominate and only the largest $(>60$ $\mathrm{mV}$ ) EJPs show the spiking time course. However, the threshold for EJP spiking decreases with development time and, by $16 \mathrm{hr}$ AEL, most (>75\%) EJPs reach the spiking threshold (Figs. $14 E$, $15 E$ ). In later stages, nearly all EJPs have the mature short time course. In addition to these "normal" EJPs, very long-duration (up to tens of seconds) EJPs continue to persist in the maturing synapse (Fig. $14 E$ ), but with decreasing frequency $(0.2 / \mathrm{min}$ at $16 \mathrm{hr}$ AEL).

\section{The mature embryonic pattern of synaptic communication (20/2I hr AEL)}

Synaptic communication in the mature embryo is characterized by frequent ( $>10 / \mathrm{min}$ ) EJCs of large ( $>25$ gluR openings) amplitude (Figs. 12,14C,15C). Though single gluR opcnings remain the most frequent event, the frequency of intermediate EJCs ( $<10$ gluR openings) has decreased drastically; most $(>80 \%)$ EJCs represent the simultaneous opening of $>10$ gluRs (Fig. 14E). All EJCs have the short $(<10 \mathrm{msec})$ mature time course; longer-time-course EJCs do not persist in the mature synapse (Fig. 14E).

Mature embryonic EJPs are brief $(<0.1 \mathrm{sec})$ overshouting $(+20-40 \mathrm{mV})$ events (Figs. $14 F, 15 F)$. Even more than the EJCs, the amplitudes of EJPs in the mature embryo tend to be consistent; most events reach the threshold of the calcium current and so reach an overshooting amplitude. All EJPs have the mature time course (Fig. 14E), and the long-time-course EJPs characteristic of early development no longer occur.
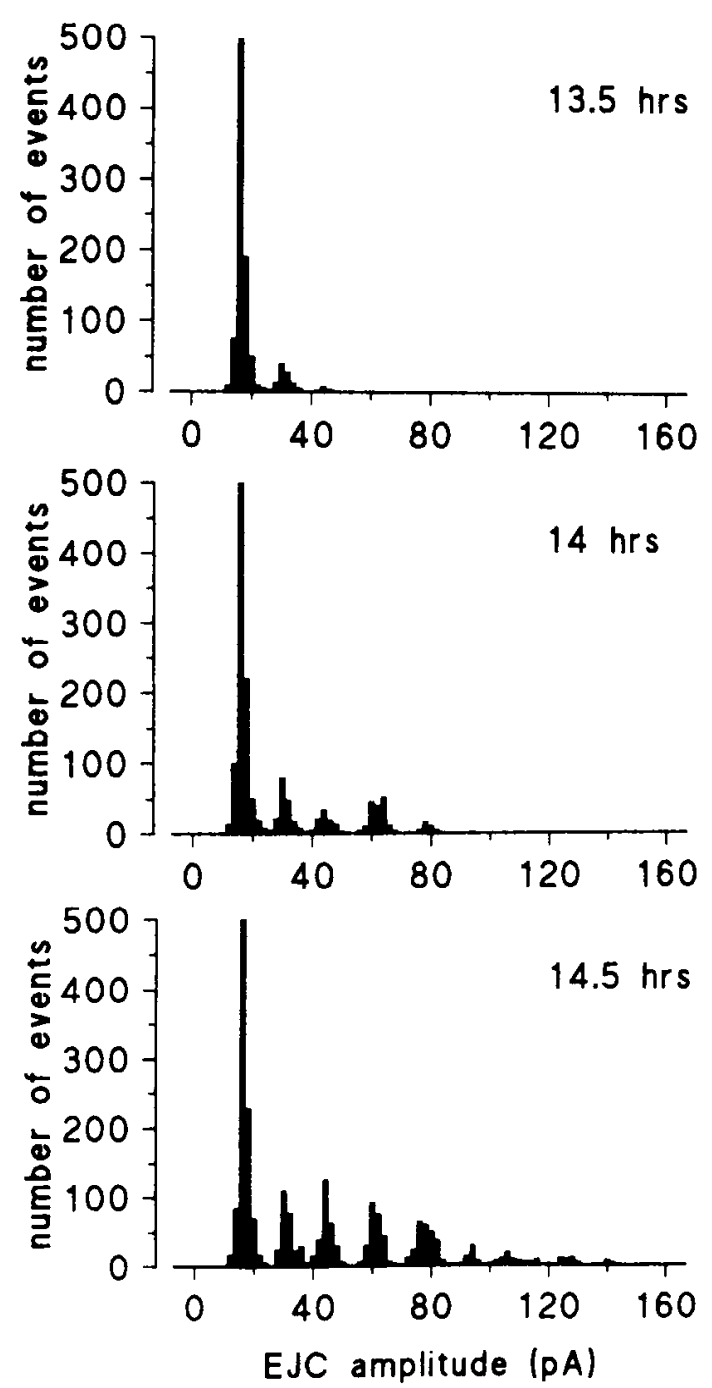

Figure 13. The amplitude distribution of endogenous EJCs during the early development of the embryonic NMJ: currents were measured in myotubes voltage clamped at $-80 \mathrm{mV}$. The amplitude of each peak correlates with multiples of single gluR channel openings. Initially (13.5 hr AEL), the vast majority of EJCs (peak $=16 \mathrm{pA}$ ) can be explained by single gluR openings, and very few multiple-opening EJCs are observed (maximum current $=50 \mathrm{pA}$ ). The mean EJC amplitude rapidly increases over the next hour as a functional neuromuscular synapse develops. By $14.5 \mathrm{hr}$ AEL, multiple-opening EJCs are common up to $120 \mathrm{pA}$, with occasional maxima up to $160 \mathrm{pA}$, but single gluR openings remain the most common event.

\section{Maturing characteristics of the embryonic synapse}

The mature NMJ must have the characteristics of a rapid, reliable, and consistent communication system. The early embryonic synapse has none of these properties and must acquire them during the course of its maturation. We have examined the development of these characteristics during synaptogenesis in two ways: (1) the development of synaptic "strength" or time course of synapse fatigue (Fig. 16) and (2) the development of synaptic "reliability" or consistency of synaptic communication (Fig. 17).

Synaptic fatigue was measured by monitoring EJC amplitude over time in response to repeated stimulation of the motor nerve. The stimulation frequency $(3 \mathrm{~Hz})$ was selected empirically, as being sufficient to differentiate fatigue levels during early synaptogenesis but below the fatigue level of the mature synapse. 


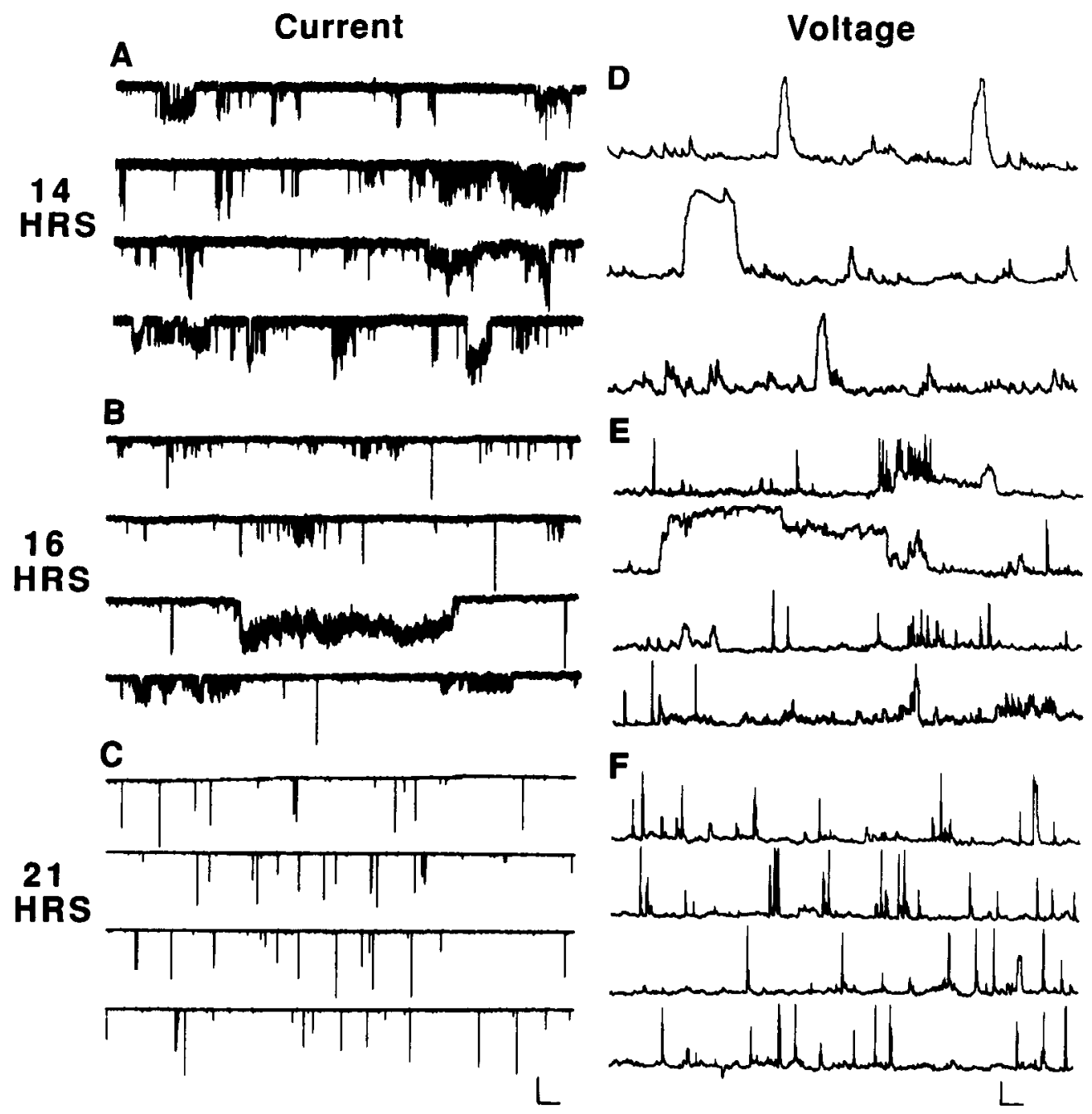

Figure 14. Endogenous synaptic communication during neuromuscular synaptogenesis. Muscle voltage-clamp ( $-60 \mathrm{mV})$ current recordings are shown in the left column; voltage recordings are shown in the right column (resting potential, $-46 \pm 6 \mathrm{mV}$ ). Representative traces from early synaptogenesis (14 hr AEL), mid synaptogenesis (16 hr AEL), and the mature embryonic NMJ (21 hr AEL, hatching) are shown. More than 10 embryos were examined at each developmental stage. $A$, Recording showing examples of the major types of currents at $14 \mathrm{hr}$ AEL. Single and double gluR openings predominate, often showing characteristic bursting activity. Multiple-opening EJCs (maximum current $=80 \mathrm{pA}$ ) are just beginning and show both the typical, brief currents $(<10 \mathrm{msec})$ as well as relatively rare, very long currents (up to seconds). $B$, Synaptic currents in the functional embryonic NMJ at $16 \mathrm{hr}$ AEL. The incidence of miniature EJCs has decreased and the incidence of macro EJCs (up to $250 \mathrm{pA}$ ) has increased. Most EJCs show the typical mature time course $(<10 \mathrm{msec})$, but very long currents (up to many seconds) are still observed at a low frequency. $C$, Synaptic currents at the mature embryonic NMJ (21 hr AEL, hatching): long time course showing an example of EJC frequency and amplitude distribution. Most currents are $>250 \mathrm{pA}$ (maximum currents $>600 \mathrm{pA}$ ) and brief $(<10 \mathrm{msec}$ ). Long-time-course currents are absent. $D$, Synaptic potentials at $14 \mathrm{hr}$ AEL. The majority of events are small, subthreshold MEJPs. Rarer, long-lasting (up to seconds), macro EJPs occur even at the earliest stages of synaptogenesis. EJPs at this period only rarely overshoot $0 \mathrm{mV}$. E, Synaptic potentials at $16 \mathrm{hr}$ AEL. The incidence of small, subthreshold MEJPs has decreased from earlier stages and the incidence of macro EJPs has incrcascd. Most of the macro EJPs show the mature phenotype in that they are brief $(<0.15 \mathrm{sec})$, "spiking," and overshoot $0 \mathrm{mV}$. However, many EJPs maintain the earlier "nonspiking" phenotype of low amplitude ( $<30 \mathrm{mV}$ ) and long time course (up to a second). Long-lasting (up to many seconds), macro EJPs continue to occur at a decreased frequency. $F$, Synaptic polentials at the mature embryonic NMJ (21 hr AEL, hatching): a long time course of EJP frequency and amplitude distribution. Potentials are brief $(<0.1 \mathrm{sec})$ and overshooting $(+20-40 \mathrm{mV})$ and show characteristics of the mature larval EJP. Calibration: $A, 25 \mathrm{pA}, 2 \mathrm{sec} ; B, 75 \mathrm{pA}, 2 \mathrm{sec} ; C, 250 \mathrm{pA}, 4 \mathrm{sec} ; D, 10 \mathrm{mV}, 3 \mathrm{sec} E, 30 \mathrm{mV}, 3 \mathrm{sec} ; F, 30 \mathrm{mV}, 3 \mathrm{sec}$.

During early synaptogenesis, repetitive stimulation of the motor nerve produced a rapid depression in the amplitude of the evoked EJCs (Fig. 16). The earliest synaptic transmission (13.5 hr AEL) fatigues extremely rapidly and is soon $(<5 \mathrm{sec})$ completely fatigued. This depression could be observed with stimulus frequencies as low as $0.1 \mathrm{~Hz}$, and was often complete within 5-10 stimuli. Half an hour later (14 hr AEL; Fig. 16), the synapse is significantly more robust, but it still fatigues rapidly and completely $(<20 \mathrm{sec})$. The newly functional NMJ $(14.5 \mathrm{hr}$ AEL; Fig.
16) is again more robust but, after initially maintaining EJC amplitude (10 sec), gradually fatigues within $40 \mathrm{sec}$. By $15 \mathrm{hr}$ AEL, full transmission is maintained longer ( $30 \mathrm{sec}$; Fig. 16), fatigue is even more gradual, and complete fatigue requires prolonged stimulation ( $>60 \mathrm{sec}$ ). From $16 \mathrm{hr}$ AEL, no significant fatigue occurs even after prolonged stimulation $(>1 \mathrm{~min}$; Fig. 16) and the synapse cannot be distinguished from the mature NMJ using these parameters. Recovery from fatigue also changes with developmental age: the earliest synapse (13.5 hr AEL) was 


\section{Current Voltage}

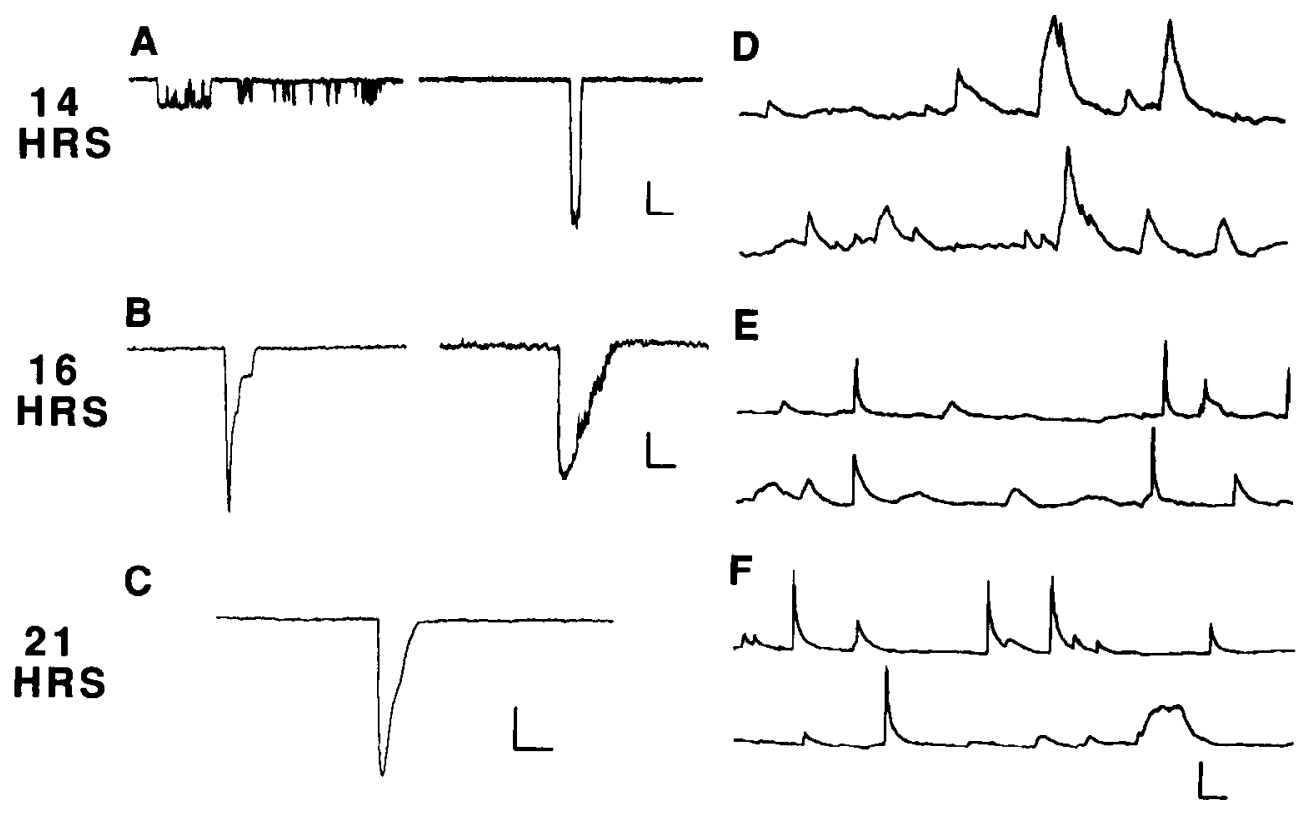

Figure 15. Development of endogenous EJCs and EJPs during synaptogenesis. Muscle voltage-clamp ( $-60 \mathrm{mV})$ current recordings are shown in the left column; voltage recordings are shown in the right column (resting potential, $-46 \pm 6 \mathrm{mV}$ ). These are representative traces from early synaptogenesis ( $14 \mathrm{hr}$ AEL), mid synaptogenesis ( $16 \mathrm{hr}$ AEL), and the mature embryonic NMJ (21 hr AEL, hatching) and should be compared to the equivalent long-time-course recordings in Figure 14. A, Example of single-gluR-opening EJCs (left) and a typical multiple-opening, macro EJC (right) at $14 \mathrm{hr}$ AEL. B, Example of a typical EJC (left) at $16 \mathrm{hr}$ AEL. On the right is an example of a long-time-course excitatory current. These currents are present during the earlier stages of synaptogenesis and increase in amplitude at the samc ratc as the charactcristic short-timc-course EJCs. $C$, Typical EJC in the mature embryo (21 hr AEL). D, Typical EJPs at $14 \mathrm{hr}$ AEL. EJPs show small amplitudes that rarely overshoot $0 \mathrm{mV}$ and have long durations $(>0.5 \mathrm{sec})$. No inhibitory potentials are observed. $E$, Typical EJPs at $16 \mathrm{hr}$ AEL. The mean EJP amplitude has increased greatly and usually overshoots O $\mathrm{mV}$. EJP duration $(<0.15 \mathrm{sec})$ is much shorter than at earlier stages. $F$, Typical EJPs at hatching $(21 \mathrm{hr}$ AEL). Average EJP amplitude has increased from earlier stages ( $16 \mathrm{hr} A E L)$, but EJP duration is similar. Calibration: $A, 20 \mathrm{pA}, 10 \mathrm{msec} ; B: l e f t, 50 \mathrm{pA}$, $10 \mathrm{msec} ;$ B.right, $50 \mathrm{pA}, 1 \mathrm{sec} ; C, 150 \mathrm{pA}, 10 \mathrm{msec} ; D, 5 \mathrm{mV}, 0.5 \mathrm{sec} ; E, 25 \mathrm{mV}, 0.25 \mathrm{sec} ; F, 30 \mathrm{mV}, 0.2 \mathrm{sec}$.

never observed to recover, the $14 \mathrm{hr}$ synapse recovered partially (50\% 2-3 min) but only rarely completely, the $14.5 \mathrm{hr}$ synapse recovered completely in $<2 \mathrm{~min}$, and the $15 \mathrm{hr}$ synapse recovered completely in $<1 \mathrm{~min}$.

The consistency of synaptic communication was measured by recording EJC amplitude in response to a repeated stimulus of the motor nerve at subfatigue levels $(1 \mathrm{~Hz})$. The immature synapse (15 hr AEL) generates EJCs of inconsistent amplitude in response to a fixed stimulus strength (Fig. 17); EJC amplitude is apparently randomly distributed from single gluR openings to the maximal EJC amplitude. In different trials, 10-25\% of stimuli failed completely to generate an EJC (Fig. 17). In contrast, in the mature embryonic synapse (21 hr AEL; Fig. 17), EJC amplitude in response to a given stimulus is much more consistent: $\operatorname{most}(>75 \%)$ EJC responses are at least $75 \%$ of the maximal EJC amplitude, and few $(<5 \%)$ give EJC responses in the lowest register ( $<25 \%$ maximum). Very few $(<2 \%)$ stimuli failed to generate a postsynaptic response (Fig. 17). The maturation of synaptic consistency is a gradual process that develops slowly throughout the later stages ( $>16 \mathrm{hr}$ AEL) of embryogenesis.

Both synaptic fatigue and inconsistency during early development reflect presynaptic immaturity. A consistent response to L-glutamatc iontophoresis at the developing synapse occurs from the earliest stages of synaptogenesis. Likewise, a synapse fully fatigued with repetitive nerve stimulation gives a full-amplitude EJC response when L-glutamate is iontophoretically applied.

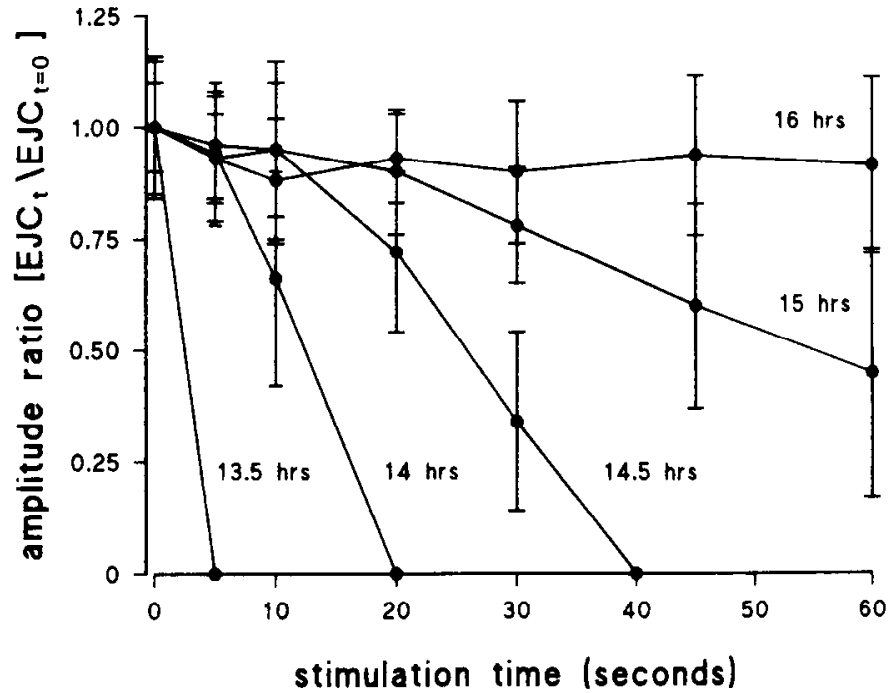

Figure 16. Development of synaptic "strength" of the maturing NMJ in the early stages of synaptogenesis. A suction electrode delivered superthreshold stimulation to the developing motor nerve at $3 \mathrm{~Hz}$, and the EJC amplitude after a period of stimulation $\left(E J C_{l}\right)$ compared to the EJC amplitude at the start $\left(E J C_{t-0}\right)$ was used to measure synaptic fatigue. At the earliest stages, the synaptic contact appeared weak and synaptic communication quickly ceased completely. However, synaptic strength rapidly increased, and within $2 \mathrm{hr}$ of the initial contact ( $16 \mathrm{hr}$ AEL) no fatigue was observed even after prolonged stimulation. Each point represents mean $\pm \mathrm{SD}(N=10$ embryos $)$. 
Figure 17. Development of the reliability of synaptic communication during the maturation of the embryonic NMJ. A suction electrode was used to deliver 100 consistent superthreshold stimulations to the developing motor nerve at $1 \mathrm{~Hz}$ (subfatigue frequency), and the EJC amplitude was measured in the myotube voltage clamped at -60 $\mathrm{mV}$. EJC amplitude is expressed as a ratio (to the maximal EJC response) to facilitate comparison of $\mathrm{EJC}$ reproducibility over development time. Early in synaptogenesis (15 hr AEL), a highly variable EJC response was obtained, with only $11 \%$ of responses reaching $75 \%$ of maximum and $15 \%$ of stimulations failing completely to elicit a response. Late in synaptogenesis $(21 \mathrm{hr}$ AEL, hatching), a much more uniform EJC response was obtained, with $72 \%$ of responses reaching $75 \%$ of maximum and no failures in synaptic communication.

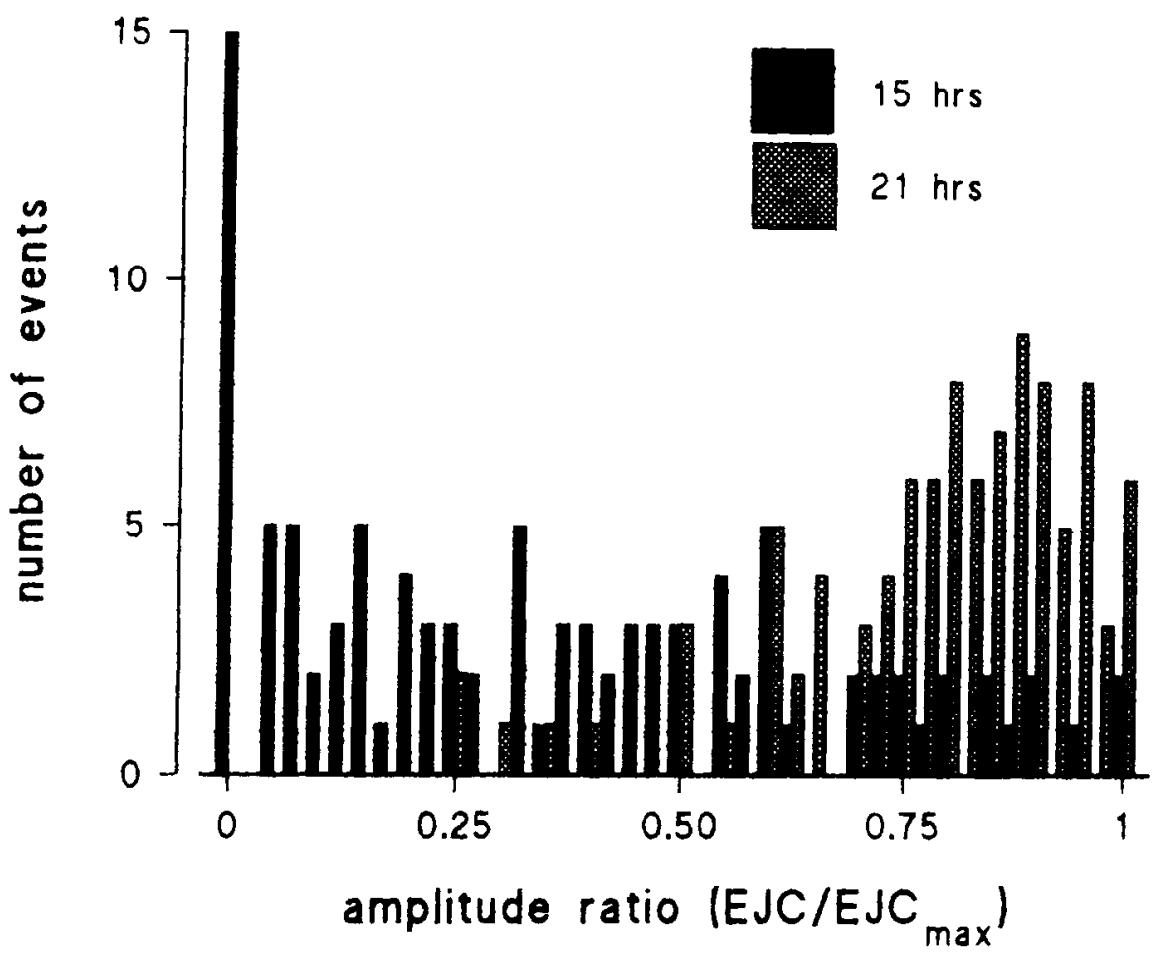

$15 \mathrm{hrs}$
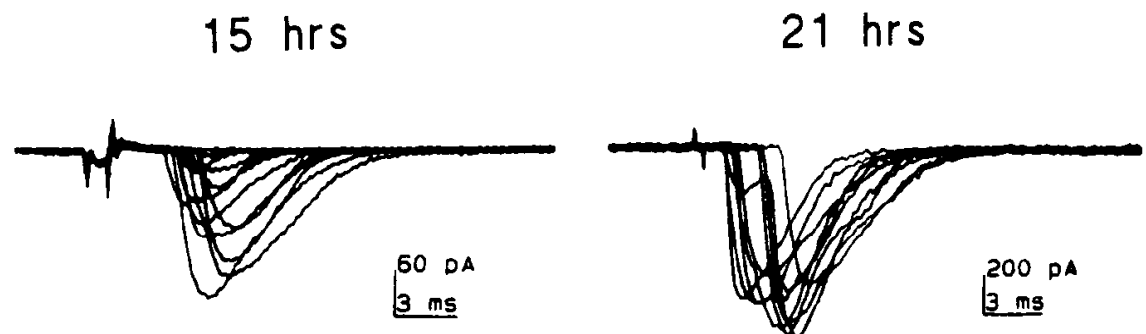

\section{Discussion}

Stages of neuromuscular synaptogenesis in Drosophila

We have examined the physiological and morphological maturation of an identified neuromuscular synapse in the Drosophila embryo. We divide neuromuscular synaptogenesis into the following sequence (Fig. 18). (1) Motor axon filopodia begin to explore the myotube surface and concurrently begin expressing neurotransmitter (12.5-13 hr AEL). (2) Myotubes uncouple to form single-cell units (13-13.25 hr AEL). (3) A small number of transmitter receptors $(<25)$ are homogeneously expressed on the myotube surface (13.25-14 hr AEL). (4) Endogenous transmitter release from pioneering growth cones is detected; nerve stimulation elicits a postsynaptic EJC response (13.25-13.75 hr AFI.). (5) Motor axon filopodia and transmitter receptors localize to the mature synaptic zone; filopodial localization is complete (14-14.5 hr AEL) in advance of receptor localization (13.75-15.5 hr AEL). (6) A functional synapse is formed (14$14.5 \mathrm{hr}$ AEL); nerve stimulation leads to muscle contraction. (7) Boutons begin to develop (14.5-15 hr AEL); NMJ attains mature morphology. (8) A second motor axon synapses on the myotube at the preestablished synaptic zone (15.5-16.5 hr AEL). (9) Vigorous neuromuscular activity, characteristic of larval locomotory movements, begins (16-16.5 hr AEL). (10) A second stage of receptor expression commences (16.5-17.5 hr AEL) and continues through to the end of embryogenesis ( $21 \mathrm{hr}$ AEL).

As far as we know, this is the first study to investigate the physiological development of the NMJ in the Drosophila embryo and so provide a basis for the comparison of physiological and morphological development (Fig. 18). Earlier work using anti-HRP staining at the light microscope level (Johansen et al., $1989 \mathrm{a}, \mathrm{b})$ and more recent studies tracing individually dye-filled motor neurons (Halpern et al., 1991; Sink and Whitington, $1991 \mathrm{a}, \mathrm{b})$ have mapped the motor neuron-muscle relationships in the developing embryo and described the time course of morphological synaptogenesis at individual synapses, including the synapse examined in this study. In general, the findings of our study (Fig. 18) confirm and extend these earlier observations. Most importantly, the timing of the initial nerve-muscle contact, the timing of filopodial exploration, the restriction of filopodia to the synaptic "cleft" between muscles 6 and 7, and the appearance of presynaptic specializations and mature morphology are in close agreement with previous studies (Halpern et al., 1991; Fig. 18).

Nevertheless, some important discrepancies remain to be resolved. For example, Johansen et al. (1989b) report that myotubes uncouple by late stage $15(\sim 12.5 \mathrm{hr}$ AEL$)$, "at least an hour before synapses are made," and so conclude that myotube 


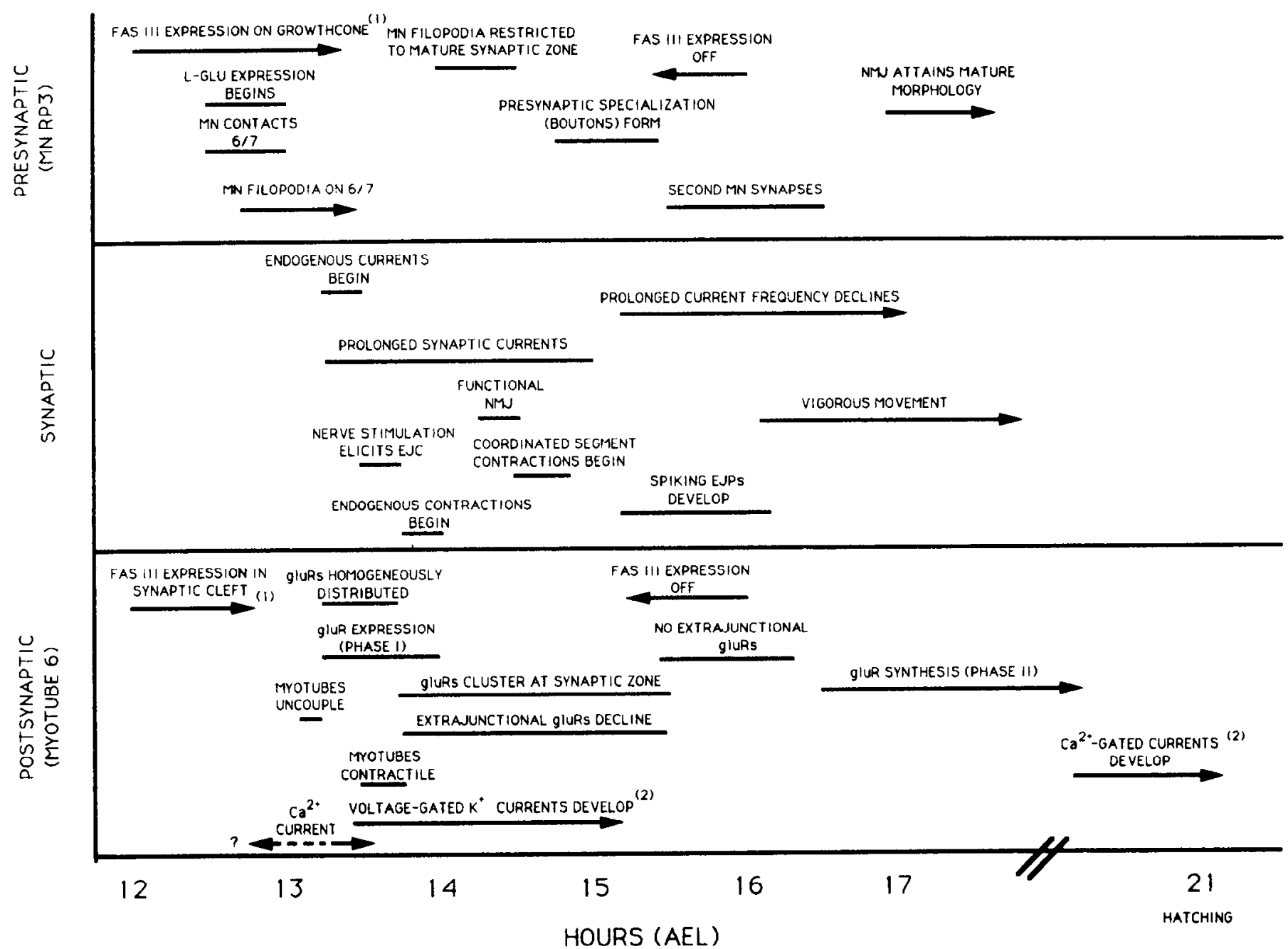

Figure 18. The time course of neuromuscular synaptogenesis in the Drosophila embryo. Development of the presynaptic (motor neuron RP3), postsynaptic (muscle 6), and synaptic (transmission at the RP3/muscle 6 NMJ) elements have been separated for clarity. Bars represent the duration of events observed; arrows indicate that the event continues in the indicated direction. All data represent results of the present study, except those indicated: (1), Halpern et al. (1991); (2), Broadie and Bate (1992).

uncoupling plays no regulative role in synaptogenesis. In contrast, we find that myotube uncoupling occurs abruptly in carly stage 16 (13-13.25 hr AEL; Fig. 18), immediately prior to imminent synapse formation. Additionally, we have shown that gluRs are first expressed immediately following myotube uncoupling (<15 min). Likewise, Johansen et al. (1989b) report that transmitter (L-glutamate) expression begins in middle to late stage 16 (14.5-15.5 hr AEL), hours after our own observation (12.5-13 hr AEL) and, indeed, more than an hour after a functional synapse has been established (Fig. 18). Such discrepancies have to do with the timing, rather than the nature, of events. However, small timing differences may lead to major differences in interpretation. In all these studies, staging has been based on morphological criteria (Campos-Ortega and Hartenstein, 1985). However, in the present work, we staged at narrow ( $<15 \mathrm{~min}$; see Materials and Methods) windows of development and then timed development from these points, whereas Johansen et al. (1989b) staged embryos dircetly by morphology at all times of development. The problem with the second tactic is that morphological markers often delineate relatively long periods ( $\gg 15 \mathrm{~min}$ ) and that there are few, if any, reliable morphological markers between the beginning of stage 16 (13 hr
AEL) and the beginning of stage 17 ( $16 \mathrm{hr}$ AEL), the $3 \mathrm{hr}$ interval during which synaptogenesis at the NMJ occurs.

\section{Properties of the embryonic and larval NMJ}

That L-glutamate, or a close analog, is the primary neurotransmitter at both the embryonic and larval NMJ on muscles 6 and 7 seems clearly established (Jan and Jan, 1976a,b; Johansen et al., 1989a,b). Although two motor neurons synapse on muscle 6 (at least in A2-A4) in late embryonic stages, both appear to be excitatory and release L-glutamate; there is no evidence for inhibitory transmission. L-Glutamate receptors in the embryo have a large unitary conductance $(200 \mathrm{pS})$ and mean open times fitting two exponential distributions (mean $=0.2$ and $2.1 \mathrm{msec}$ ) with a very few longer events (mean $=11.8 \mathrm{msec}$ ). Whether this reflects a heterogeneous population of receptors or one receptor with several open states could not be resolved in the whole-cell configuration employed. However, preliminary evidence with cell-attached patch-clamp suggests a single receptor type with multiple open states depending on L-glutamate concentration.

L-Glutamate receptors are localized to the synaptic zone during the early stages of synaptogenesis, and no extrajunctional receptors are present in later embryonic stages (>16 hr AEL). 
This localization appears to be via lateral movement of preexisting gluRs during early synaptogenesis, but may involve other mechanisms during later development. These findings are in agreement with studies in the larva (Jan and Jan, 1976a,b; Johansen et al., 1989a) that have shown that L-glutamate chemosensitivity is confined to within a few microns of the presynaptic nerve terminals (but see Delgado et al., 1989). These findings differentiate Drosophila from grasshoppers, which have distinct populations of junctional and extrajunctional gluRs (Dudel et al., 1988), but are similar to results at the vertebrate NMJ (reviewed in Schuetze, 1986).

The large conductance of the gluR channel, coupled with the high input resistance of the embryonic myotube, means that the opcning of a single channel results in a miniature EJP (MEJP) of several millivolts (mean $=2.8 \mathrm{mV}$ ). Thus, the opening of a small number of gluRs is sufficient to reach the threshold of the inward calcium current (see following companion article, Broadie and Bate, 1992) and so elicit a muscle contraction. This explains how a small number of receptors $(<25)$ can establish a functional NMJ in early embryonic development. The MEJPs at the embryonic synapse are much larger than MEJPs in the larval muscle (mean $=0.6 \mathrm{mV}$; Jan and Jan, 1976a,b) and are as large as, or larger than, MEJPs at vertebrate NMJs (Saltpeter, 1987). However, MEJPs in the mature and immature synapse appear to reflect very different events. In the mature synapse, both in Drosophila and vertebrate systems, an MEJP results from a release of a transmitter vesicle and subsequent opening of many receptors; in the Drosophila embryo, an MEJP results from a single gluR channel opening. Thus, the opening of a single gluR channel at the immature NMJ has the same, or greater, functional consequence as the release of a transmitter vesicle and subsequent opening of many receptors at the mature NMJ.

As at the vertebrate $\mathrm{NMJ}$, we find that transmission at the embryonic Drosophila NMJ is based on quantal units. However, the quantum at the immature NMJ is postsynaptic, not presynaptic, being based on the opening of a single gluR channel rather than the release of a vesicle of transmitter. Furthermore, because of the very low number of gluRs during embryogenesis, receptor numbers are always the limiting factor in synaptic transmission. This restricts our ability to determine the nature of presynaptic transmitter release at the developing synapse: the release of even very small amounts of transmitter (less than one vesicle) is likely to open all the receptors clustered at the postsynaptic release site. [This is assuming that a vesicle contains on the order of several thousand transmitter molecules as has been reported in other systems (Saltpeter, 1987)]. That not all receptors are activated by a nerve impulse suggests that either (1) very small amounts of transmitter are actually released during early synaptogenesis (less than vesicle per nerve impulse; i.e., transmitter release is nonquantal) or (2) the small number of receptors are distributed over a wide area of possible transmitter release and so are not available to the released transmitter. In the second explanation, the release of transmitter at any one site happens only with a certain probability with each nerve impulse, and only the receptors within this limited area are activated before the transmitter is taken up or diffuses away. It is possible that these localized transmitter release sites may prefigure the early development of the specialized presynaptic release sites, or boutons, seen in the mature larva.

We observe two distinct types of excitatory currents during synaptogenesis: (1) a current with a short time course $(<10$ msec) that resembles the mature EJC in the larva and (2) currents with very long time courses (usually hundreds of milliseconds but up to seconds) peculiar to carly synaptogenesis. Similar types of currents have been described during the early phases of NM J formation in vertebrate systems (Poo et al., 1985) and, with their superior assay system, were shown to represent quantal and nonquantal transmitter release, respectively. It is tempting to speculate that there is a similar distinction during Drosophila synaptogenesis. The alternative is to suggest that the early synapse is capable of a continuous release of synaptic vesicles for up to many seconds. However, the rapidity of fatigue at the early synapse makes such an explanation unlikely. It is more probable that the developing motor terminal is capable of the long-term nonquantal release of L-glutamate, perhaps in response to a specific signal, as well as the quantal release of transmitter vesicles in response to a nerve impulse. Thus, the short EJCs would represent the development of synaptic communication, while the prolonged, nonquantal release may play some developmental role during early synaptogenesis or, alternatively, be a byproduct of early development that plays no significant physiological rolc.

The early synapse shows inconsistent communication properties that fatigue rapidly. Given that the postsynaptic L-glutamate receptors do not inactivate rapidly, and a fully fatigued synapse will still respond to iontophoretically applied L-glutamate with a full-amplitude EJC, both inconsistency and fatigue must arise from presynaptic immaturity rather than some postsynaptic mechanism. A likely hypothesis is that only a small amount of transmitter is available at the presynaptic release sites during early synaptogenesis and that rapid stimulation quickly exhausts this small transmitter store. Likewise, the immaturity of transmitter release sites could explain the inconsistency of synaptic communication. A given nerve impulse would cause release of only those synaptic vesicles present at the active presynaptic membrane, and the number of vesicles is likely to be variable at a developing release site. Studies in vertebrate preparations (Buchanan et al., 1989; Lupa and Hall, 1989) have shown that while transmitter vesicles are present during the first hours of synaptic development at the NMJ, the vesicles are distributed throughout the cytoplasm and there is little differentiation of release sites, or active zones, with characteristic clusters of synaptic vesicles. It seems likely that a similar immaturity underlies the inconsistent communication properties and rapid fatigue during early synaptogenesis in Drosophila.

\section{Mechanisms of neuromuscular synaptogenesis}

The aim of this work has been to characterize and provide a developmental timetable of synaptogenesis at the NMJ in Drosophila, and so establish the basis for investigation of the mechanisms underlying synaptic development. Several of our observations lend themselves to possible mechanistic interpretations. For example, (1) transmitter is expressed at the time of initial nerve-muscle contact, (2) the myotubes uncouple soon (within several minutes) after motor neuron filopodia begin growing on the myotube surface, (3) transmitter receptors are expressed immediately (within several minutes) following myotube uncoupling, (4) receptor localization closely follows motor neuron filopodia localization at the developing synaptic zone, and (5) the second stage of receptor expression coincides with the arrival of a second motor axon and the onset of vigorous muscle activity. Whether these and similar (see Fig. 18) correlations reveal significant interactions is currently under investigation. 
Of course, temporal correlations are insufficient evidence to propose developmental interactions, but they do eliminate several possible formative mechanisms. For example, studies in vertebrate systems (Poo et al., 1985) have demonstrated that neural growth cones release transmitter during pathfinding, and it has been suggested that neuronal pathfinding interactions between growth cones and their environment may be mediated via transmitter-receptor interactions. This is clearly not the case during peripheral pathfinding in Drosophila, as neither transmitter nor receptor is present until synaptogenesis begins. Likewise, transmitter-receptor interactions cannot be involved in growth cone-target muscle recognition because both elements are absent during the critical choice period. Furthermore, transmitter-receptor interactions cannot play a role in the earliest stages of synaptogenesis at the NMJ, because the motor neuron filopodia are already restricted to the synaptic cleft before the appearance and localization of the transmitter receptors. These observations are in agreement with recent work in the zebrafish (Westerfield et al., 1990) that shows that completely normal NMJs are established in a mutant (nic-I) that lacks all AChRs. Of course, these arguments exclude only the primary excitatory chemical (L-glutamate or ACh) as a transmitter in such putative interactions; there could be interactions occurring via other transmitter-receptor pathways.

In addition to transmitter-receptor interactions, it has long been suggested that selective adhesion forms the basis of neuronal pathfinding in many other systems (for review, see $\mathrm{Ru}$ tishauser and Jessel, 1988) and, more recently, in the Drosophila embryo (Grenningloh et al., 1990). An extension of this hypothesis argues that selective adhesion may form the basis of nerve-muscle recognition and, indeed, define the mature synaptic contact area during synaptogenesis (Landmesser et al., 1990). Analysis of expression patterns (Johansen et al., 1989b; Halpern et al., 1991) has suggested that such selective adhesion might explain the stereotyped progression of synapse development of RP3 and muscles 6 and 7. These studies have shown that fasciclin III (fas III), a molecule known to mediate homeophilic aggregation (Snow et al., 1989) and implicated in early neural fasciculation, is transiently expressed on both the RP3 growth cone and the synaptic cleft of muscles 6 and 7 from 12 $16 \mathrm{hr}$ AEL (Halpern et al., 1991). That fas III, and/or similar molecules, may initiate and, perhaps, guide motor neuron outgrowth at this synapse agrees well with our data (Fig. 18); expression begins immediately before the initial nerve-muscle contact and stops when the robust, functional NMJ has been established. Such an adhesion model could explain why filopodial processes are restricted during development to the fas III-expressing synaptic cleft and quickly confined to the mature synaptic zonc.

We find that motor neuron filopodia are located in the synaptic cleft between muscles 6 and 7 from the earliest stages of neuron-muscle contact, and that the filopodia are wholly restricted to the mature synaptic zone prior to localization of the postsynaptic receptors. These observations exclude the possibility that synaptic sites are prefigured by clusters of postsynaptic receptors, as has been proposed in some vertebrate preparations. On the other hand, it seems probable that receptors are localized to synaptic sites through the action of the motor neuron. Indeed, many studies in vertebrates (reviewed in Saltpeter and Loring, 1985; Rochlin and Peng, 1990) have shown that motor neurons are able to induce clustering of postsynaptic receptors. This clustering has been shown to result from motor neuron activity
(Rochlin and Peng, 1990), in part at least, probably mediated via transmitter release (Zhu and Peng, 1988). It is possible that the prolonged hursts of transmitter release seen in early synaptogenesis (as discussed above) may be involved in gluR clustering. Other studies (Magill-Solc and McMahan, 1990; Wallace, 1991) have revealed two presynaptic signaling molecules, agrin and ARIA, which induce postsynaptic receptor clustering and synthesis, respectively. The timing of receptor localization in Drosophila strongly suggests that the motor neuron may signal gluR aggregation in a similar fashion, though the signaling mechanism remains to be elucidated.

Cell-cell signaling from both the presynaptic and postsynaptic cells is likely to underlie the establishment of the precise neuromuscular synapse. The work presented here on the morphological and physiological interactions of two identified synaptic partners during synapse development provides the groundwork for an cxperimental analysis of mechanisms controlling synaptogenesis. The experimental advantages of the relatively simple Drosophila embryonic NMJ will enable us extend our analysis beyond the cellular level and identify the genes regulating synapse formation for the first time.

\section{References}

Allen F, Warner A (1991) Gap junctional communication during neuromuscular junction formation. Neuron 6:101-111.

Anderson M, Cohen M (1977) Nerve-induced and spontaneous redistribution of acetylcholine receptors on cultured muscle cells. J Physiol (Lond) 268:757-773.

Anderson M, Halpern M, Keshishian H (1988) Identification of the neurotransmitter proctolin in Drosophila larvae: characterization of muscle fiber-specific neuromuscular endings. J Neurosci 8:242-255.

Balicegordon R, Lichtman J (1990) In vivo visualization of the growth of presynaptic and postsynaptic elements of neuromuscular junctions in the mouse. J Neurosci 10:894-908.

Bate M (1990) The embryonic development of larval muscles in Drosophila. Development 110:791-804.

Bennet M (1983) Development of neuromuscular synapses. Physiol Rev 63:915-1048.

Blackshaw S, Warner A (1976) Low resistance junctions between mesoderm cells during development of trunk muscles. J Physiol (Lond) 245:209-230.

Broadie K, Skaer H, Bate M (1992) Wholc-cmbryo culturc of Drosophila: development of embryonic tissues in vitro. Rouxs Arch Dev Biol, in press.

Broadie KS, Bate M (1993) Development of larval muscle properties in the embryonic myotubes of Drosophila melanogaster. J Neurosci 13:167-180.

Buchanan J, Sun Y-a, Poo M-m (1989) Studies of nerve-muscle interactions in Xenopus cell culture: fine structure of early functional contacts. J Neurosci 9:1540-1554.

Bursztajn S, Berman S, Gilbert W (1989) Differential expression of acetylcholine receptor messenger RNA in nuclei of culture muscle cells. Proc Natl Acad Sci USA 86:2928-2932.

Campos-Ortega J, Hartenstein V (1985) The embryonic development of Drosophila melanogaster. Berlin: Springer.

Currie D, Milner M, Evans C (1988) The growth and differentiation in vitro of leg and wing imaginal disc cells from Drosophila melanogaster. Development 102:805-814.

Delgado R, Barla R, Latorre R, Labarca P (1989) L-Glutamate activates excitatory and inhibitory channels in Drosophila larval muscle. FEBS Lett 243:337-342.

Dennis M, Ziskind-Conhaim L, Harris A (1981) Development of neuromuscular junctions in rat embryos. Dev Biol 81:266-279.

Dudel J, Franke C. Hatt H, Ramsey R, Usherwood P (1988) Rapid inactivation and desensitization by glutamate of excitatory, cationselective channels in locust muscle. Neurosci Lett 88:33-38.

Evers J, Laser M, Sun Y-n, Xie Z-p, Poo M-m (1989) Studies of nervemuscle interactions in Xenopus cell culture: analysis of early synaptic currents. J Neurosci 9:1523-1539. 
Ganetzky B, Wu C-F (1986) Neurogenetics of membrane excitability in Drosophila. Annu Rev Genet 20:13-44.

Grenningloh G, Bieber A, Rehm E, Snow P, Traquina Z, Hortsch M, Patel N, Goodman C (1990) Molecular genetics of neuronal recognition in Drosophila: evolution and function of immunoglobulin superfamily adhesion molecules. Cold Spring Harbor Symp Quant Biol 50:327-340.

Halpern M, Chiba A, Johansen J, Keshishian H (1991) Growth cone behavior underlying the development of stereotypic synaptic connections in Drosophila embryos. J Neurosci 11:3227-3238.

Hamill O, Marty A, Neher E, Sakmann B, Sigworth F (1981) Improved patch-clamp techniques for high-resolution current recording from cells and cell-free membrane patches. Pfluegers Arch 391:85-100.

Jackson H, Usherwood P (1988) Spider toxins as tools for dissecting elements of excitatory amino acid transmission. Trends Neurosci 11 ; 278-283.

Jan L, Jan Y (1976a) Properties of the larval neuromuscular junction in Drosophila melanogaster. J Physiol (Lond) 262:189-214.

Jan L, Jan Y (1976b) L-Glutamate as an excitatory transmitter at the Drosophila larval neuromuscular junction. J Physiol (Lond) 262:215236.

Jan L, Jan Y (1982) Antibodies to horseradish peroxidase as specific neuronal markers in Drosophila and grasshopper embryos. Proc Natl Acad Sci USA 72:2700-2704.

Johansen J, Halpern M, Johansen K, Keshishian H (1989a) Stereotypic morphology of glutamatergic synapses on identified muscle cells of Drosophila larvae. J Neurosci 9:710-725.

Johansen J, Halpern M, Keshishian H (1989h) Axonal guidance and the development of muscle fiber-specific innervation in Drosophila embryos. J Neurosci 9:4318-4332.

Korn S, Mary A, Connor J, Horn R (1991) Perforated patch recording. Methods Neurosci 4:264-373.

Kullberg R, Lentz T, Cohen M (1977) Development of the myotomal neuromuscular junction in Xenopus laevis: an electrophysiological and fine-structural study. Dev Biol 60:101-129.

Landmesser L, Dahm L, Tang J, Rutishauser U (1990) Polysialic acid as a regulator of intramuscular nerve branching during embryonic development. Neuron 4:655-667.

Lieth E, Cardasis C, Fallon J (1992) Muscle-derived agrin in cultured myotubes-expression in the basal lamina and at induced acetylcholine-receptor clusters. Dev Biol 149:41-54.

Lupa M, Hall Z (1989) Progressive restriction of synaptic vesicle protein to the nerve terminal during development of the neuromuscular junction. J Neurosci 9:3937-3945.

Magill-Solc C, McMahan U (1990) Agrin-like molecules in motor neurons. J Physiol (Paris) 84:78-81.

Marty A, Neher E (1983) Tight-seal whole-cell recording. In: Singlechannel recording (Sakmann B, Neher E, eds), pp 107-121. New York: Plenum.

Poo M-m, Sun Y-a, Young S (1985) Three types of transmitter release from embryonic neurons. J Physiol (Paris) 80:283-289.
Rochlin M, Peng H (1990) The influence of AChR clustering stimuli $n n$ the formation and maintenance of $A C h R$ clusters induced by polycation-coated beads in Xenopus muscle cells. Dev Biol 140:2740.

Rutishauser U, Jessel T (1988) Cell adhesion molecules in vertebrate ncural devclopment. Physiol Rev 68:819-857.

Saltpeter M (1987) The vertebrate neuromuscular junction. New York: Liss.

Saltpeter M, Loring R (1985) Nicotinic acetylcholine receptors in vertebrate muscle: properties, distribution and neural control. Prog Neurobiol 25:297-325.

Sanes D, Poo M-m (1988) In vitro analysis of specificity during nervemuscle synaptogenesis. In: Ciba Foundation symposium 138, Plasticity of the neuromuscular system, pp 116-130. Chichester: Wiley.

Schuetze S (1986) Embryonic and adult acetylcholine receptors: molecular basis of developmental changes in ion channel properties. Trends Neurosci 9:111-113.

Shadiack A, Nitkin R (1991) Agrin induces alpha-actinin, filamin, and vinculin to co-localize with AChR clusters on cultured chick myotubes. J Neurobiol 22:617-628.

Shields G, Sang J (1977) Improved medium for culture of Drosophila embryonic cells. Drosophila Inform Serv 52:161.

Sink H, Whitington P (1991a) Pathfinding in the central nervous system and periphery by identified embryonic Drosophila motor axons. Development 112:307-316.

Sink H, Whitington P (1991b) Location and connectivity of abdominal motoneurons in the embryo and larva of Drosophila melanogaster. $J$ Neurobiol 22:298-311.

Snow P, Bieber A, Goodman C (1989) Fasciclin III: a novel homophilic adhesion molecule in Drosophila. Cell 59:313-323.

Tse F, Marin L, Jahromi S, Atwood H (1991) Variations in terminal morphology and presynaptic inhibition at crustacean neuromuscular junctions. J Comp Neurol 304:135-146.

Wallace B (1991) The mechanism of agrin-induced acetylcholine-receptor aggregation. Philos Trans R Soc Lond [Biol] 331:273-280.

Westerfield M, Liu D, Kimmel C, Walker C (1990) Pathfinding and synapse formation in a zebrafish mutant lacking functional acetylcholine receptors. Neuron 4:867-974.

Wigston D (1990) Repeated in vivo visualization of neuromuscular junctions in adult mouse lateral gastrocnemius. J Neurosci 10:17531764.

Yoshikami D, Okun L (1984) Staining of living presynaptic nerve terminals with selective fluorescent dyes. Nature 310:53-56.

Zhong Y, Wu C-F (1991) Altered synaptic plasticity in Drosophila memory mutants with a defective cyclic AMP cascade. Science 251: 198-201.

Zhu D, Peng H (1988) Increase in intracellular calcium induced by the polycation-coated latex bead: a stimulus that causes post-synaptictype differentiation in cultured Xenopus muscle cells. Dev Biol 126: 63-70. 